\title{
Constraints from Dust Mass and Mass Accretion Rate Measurements on Angular Momentum Transport in Protoplanetary Disks
}

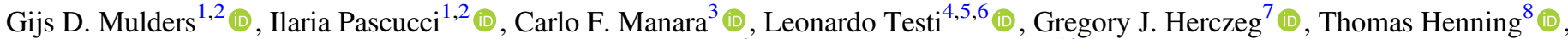 \\ Subhanjoy Mohanty ${ }^{9}$ (i), and Giuseppe Lodato ${ }^{10}$ \\ ${ }^{1}$ Lunar and Planetary Laboratory, University of Arizona, Tucson, AZ 85721, USA; mulders@1pl.arizona.edu \\ ${ }^{2}$ Earths in Other Solar Systems Team, NASA Nexus for Exoplanet System Science, USA \\ ${ }^{3}$ Scientific Support Office, Directorate of Science, European Space Research and Technology Centre (ESA/ESTEC), Keplerlaan 1, \\ 2201 AZ Noordwijk, The Netherlands \\ ${ }^{4}$ European Southern Observatory, Karl-Schwarzschild-Strasse 2, D-85748 Garching bei München, Germany \\ ${ }^{5}$ INAF-Arcetri, Largo E. Fermi 5, I-50125 Firenze, Italy \\ ${ }^{6}$ Gothenburg Center for Advance Studies in Science and Technology, Chalmers University of Technology and University of Gothenburg, \\ SE-412 96 Gothenburg, Sweden \\ ${ }^{7}$ Kavli Institute for Astronomy and Astrophysics, Peking University, Yi He Yuan Lu 5, Haidian Qu, 100871 Beijing, China \\ ${ }_{8}^{8}$ Max Planck Institute for Astronomy, Königstuhl 17, D-69117 Heidelberg, Germany \\ ${ }^{9}$ Imperial College London, 1010 Blackett Lab, Prince Consort Road, London SW7 2AZ, UK \\ ${ }^{10}$ Dipartimento di Fisica, Università Degli Studi di Milano, Via Celoria, 16, Milano, I-20133, Italy \\ Received 2017 April 23; revised 2017 August 17; accepted 2017 August 24; published 2017 September 20
}

\begin{abstract}
In this paper, we investigate the relation between disk mass and mass accretion rate to constrain the mechanism of angular momentum transport in protoplanetary disks. We find a correlation between dust disk mass and mass accretion rate in Chamaeleon I with a slope that is close to linear, similar to the one recently identified in Lupus. We investigate the effect of stellar mass and find that the intrinsic scatter around the best-fit $M_{\mathrm{dust}}-M_{\star}$ and $\dot{M}_{\text {acc }}-M_{\star}$ relations is uncorrelated. We simulate synthetic observations of an ensemble of evolving disks using a Monte Carlo approach and find that disks with a constant $\alpha$ viscosity can fit the observed relations between dust mass, mass accretion rate, and stellar mass but overpredict the strength of the correlation between disk mass and mass accretion rate when using standard initial conditions. We find two possible solutions. In the first one, the observed scatter in $M_{\text {dust }}$ and $\dot{M}_{\text {acc }}$ is not primordial, but arises from additional physical processes or uncertainties in estimating the disk gas mass. Most likely grain growth and radial drift affect the observable dust mass, while variability on large timescales affects the mass accretion rates. In the second scenario, the observed scatter is primordial, but disks have not evolved substantially at the age of Lupus and Chamaeleon I owing to a low viscosity or a large initial disk radius. More accurate estimates of the disk mass and gas disk sizes in a large sample of protoplanetary disks, through either direct observations of the gas or spatially resolved multiwavelength observations of the dust with ALMA, are needed to discriminate between both scenarios or to constrain alternative angular momentum transport mechanisms such as MHD disk winds.
\end{abstract}

Key words: accretion, accretion disks - planets and satellites: formation - protoplanetary disks - stars: low-mass

Supporting material: machine-readable table

\section{Introduction}

Gas-rich dusty disks around pre-main-sequence stars are the sites of planet formation; hence, their evolution and dispersal affect when and what types of planets can form. Observations have established that accretion of disk gas onto the star is a ubiquitous phenomenon (e.g., Hartmann et al. 2016) that appears to drive the early evolution of protoplanetary disks (e.g., Alexander et al. 2014). Yet, the physical mechanism by which gas loses angular momentum and accretes is still hotly debated (see, e.g., Armitage 2011; Turner et al. 2014, for comprehensive reviews on the topic).

The prevailing view has been that turbulence in disks transports angular momentum outward, enabling disk material to flow radially inward. The most common approach to parameterize the strength of turbulence is to assume the scaling relation between the viscosity, $\nu$, and the disk properties proposed by Shakura \& Sunyaev (1973):

$$
\nu=\alpha c_{s} h
$$

in the notation of Pringle (1981), where $c_{s}$ is the sound speed, $h$ is the disk scale height, and $\alpha$ is a dimensionless parameter that represents the efficiency of angular momentum transport. When assuming that $\alpha$ is independent of time and radius, it is possible to construct models that describe the disk thermal structure and its evolution (e.g., Stepinski 1998b; Armitage 2011). The simplicity of these constant- $\alpha$ disk models has led to their widespread use both to predict the evolution and dispersal of protoplanetary disks (e.g., Alexander et al. 2006; Owen et al. 2011) and dust evolution (e.g., Birnstiel et al. 2012) and to connect disk evolution to planet formation and composition (e.g., Mordasini et al. 2009; Cridland et al. 2017). Another approach is to assume that the turbulenceinduced viscosity is time independent and scales radially with a power law, in which case self-similar solutions can be developed to analytically describe the disk evolution (e.g., Lynden-Bell \& Pringle 1974).

A different approach is to compute the viscosity that arises from some turbulent process and then relate it to $\alpha$ through the framework discussed above. Magnetorotational instability (MRI; Balbus \& Hawley 1991) is thought to be the leading mechanism to drive turbulence in disks, while other instabilities such as gravitational (e.g., Kratter \& Lodato 2016) or hydrodynamic (e.g., Malygin et al. 2017) are likely to play a 
minor role (e.g., Turner et al. 2014). Global magnetohydrodynamic (MHD) simulations of accretion disks in the ideal limit support this view and find a rate of angular momentum transport $\alpha$ of $10^{-3}-10^{-2}$ with modest radial variations (Flock et al. 2011, 2013).

However, it was long realized that MRI cannot operate in the entire disk, especially at intermediate radii $(\sim 1-10 \mathrm{au})$, where the midplane is cool and shielded from ionizing radiation. This led to the development of layered disks in which accretion occurs primarily through an active ionized surface (Gammie 1996). In the dead zone, turbulent stress decreases by orders of magnitude (e.g., Flock et al. 2017) and the assumption that $\alpha$ is a global constant breaks down (e.g., Mohanty et al. 2013). The inclusion of nonideal MHD effects further complicates this picture, as simulations suggest that accretion is shut off even in the disk surface (e.g., Bai \& Stone 2013; Kunz \& Lesur 2013; Gressel et al. 2015) but strong winds develop that extract angular momentum and enable disk accretion. If these winds dominate the angular momentum transport, the evolution of protoplanetary disks cannot be described by $\alpha$ disk models (e.g., Kalyaan et al. 2015; Bai 2016).

Direct observational estimates of the turbulent motions of gas are only available for a few disks (Teague et al. 2016; Flaherty et al. 2017). In the context of $\alpha$ disk models, the observed disk masses, sizes, mass accretion rates, and lifetimes suggest $\alpha$ of order $\sim 0.01$ (Hartmann et al. 1998; Stepinski 1998a; Andrews et al. 2010) or smaller (Rafikov 2017). However, the steep mass accretion rate-stellar mass relation $\left(\dot{M}_{\text {acc }} \sim M_{\star}{ }^{2}\right.$; e.g., Natta et al. 2006; Fang et al. 2009; Alcalá et al. 2014) remains challenging to explain. Hartmann et al. (2006) suggest that the steepness results from disks around very low mass stars being less massive, fully magnetically active, and as such having viscously evolved substantially. On the other hand, Ercolano et al. (2014) propose that the relation is caused by a specific disk dispersal mechanism, stellar X-raydriven photoevaporation. Interestingly, Alexander \& Armitage (2006) and Dullemond et al. (2006) point out that the $\dot{M}_{\mathrm{acc}}-M_{\star}$ relation may not reflect disk evolution, but rather the initial conditions of star formation, specifically the disk size.

The zeroth-order expectation of viscously evolved disks is that their mass accretion rate correlates linearly with disk mass (e.g., Dullemond et al. 2006; Rosotti et al. 2017). Recent surveys of nearby star-forming regions are enabling us for the first time to test this prediction on statistically significant samples where mass accretion rates and disk masses are available for the same objects. Manara et al. (2016b) used 66 objects from the $\sim 1-3$ Myr old Lupus star-forming region with mass accretion rates homogeneously computed from VLT/XShooter spectra and disk masses from submillimeter continuum emission from the Atacama Large Millimeter/submillimeter Array (ALMA). The relation between mass accretion rates and dust disk masses is found to be roughly consistent with viscously evolved disks.

Here, we expand on this study by combining the ALMA and $\mathrm{X}$-Shooter surveys of disks in the Lupus and Chamaeleon I star-forming regions, thus more than doubling the sample of Manara et al. (2016b) (Section 2). First, we investigate the relation between dust mass, mass accretion rate, and stellar mass (Section 3). Then, we simulate a population of constant- $\alpha$ disks using a Monte Carlo approach and quantify how the observed dust mass and mass accretion rate deviate from the simulated ones (Section 4). Finally, we discuss the implications of our results and what observations/analysis should be carried out to further constrain the angular momentum transport in protoplanetary disks (Section 5).

\section{Homogeneous Analysis of Stellar and Disk Properties}

We perform a homogeneous analysis of the dust disk mass, mass accretion rate, and stellar mass in the Chamaeleon I and Lupus star-forming regions. All observational data used in this analysis were previously published; the ALMA data surveys of disk masses were presented by Ansdell et al. (2016) and Pascucci et al. (2016); the X-Shooter surveys of mass accretion rates were presented by Alcalá et al. $(2014,2017)$ and Manara et al. (2014, 2016a, 2017). The dust mass and mass accretion in Lupus were jointly analyzed by Manara et al. (2016b).

We use the stellar properties derived by Pascucci et al. (2016) using the Baraffe et al. (2015) and (nonmagnetic) Feiden (2016) evolutionary tracks to achieve a homogeneous data set for both star-forming regions, and we recalculate mass accretion rates from the accretion luminosity. We also use the dust masses for Lupus derived by Pascucci et al. (2016) for consistency. All data used in this paper, including error bars and upper limits, are presented in Table 1.

\subsection{Chamaeleon I}

The data set of Chamaeleon I is based on the ALMA survey presented by Pascucci et al. (2016) and the X-Shooter survey presented by Manara et al. (2016a, 2017).

Dust masses are taken from the ALMA continuum survey at $887 \mu \mathrm{m}$ from Pascucci et al. (2016). Of the 93 sources, 66 are detected $(>3 \sigma)$ and 27 have upper limits. We adopt the dust masses derived with a temperature of $T_{\text {dust }}=20 \mathrm{~K}$. Although the dust temperature may scale with stellar luminosity and hence mass (Andrews et al. 2013), this assumption is dependent on how the disk outer radius scales with stellar mass (Hendler et al. 2017). Using a stellar-mass-independent temperature avoids introducing a correlated error between dust mass and stellar mass. While a disk temperature that decreases with stellar mass flattens the $M_{\text {dust }}-M_{\star}$ relation (e.g., Pascucci et al. 2016) and weakens the $M_{\text {dust }}-\dot{M}_{\text {acc }}$ relation (Manara et al. 2016 b), the intrinsic scatter around the $M_{\text {dust }}-\dot{M}_{\text {acc }}$ relation remains unchanged. Hence, we focus our analysis on understanding the scatter more than the slope of the $M_{\text {dust }}-\dot{M}_{\text {acc }}$ relation. After removing stars without a mass accretion rate measurement from the X-Shooter survey (see below), the sample of stars discussed here has 63 detections with ALMA in dust continuum and 21 upper limits (Figure 1).

Accretion luminosities were taken from the $\mathrm{X}$-Shooter survey presented by Manara et al. (2017). Of the 93 sources targeted with X-shooter, 9 sources have no accretion measurement for reasons listed in the last column of Table 1 . We do not remove known transition disks from the sample, as they do not appear to be outliers based on their dust masses and mass accretion rates (see section Section 3.1.1). The sample consists of 67 accreting sources and 15 dubious accretors. Dubious accretors are stars with an emission-line strength consistent with chromospheric activity (see Manara et al. 2017, for details). We will display them as upper limits in all figures and verify throughout the paper that treating them as upper limits or detections does not influence our results. 
Table 1

Stellar and Disk Properties for Chamaeleon I and Lupus

\begin{tabular}{|c|c|c|c|c|c|c|c|c|c|c|c|c|c|c|c|}
\hline $\begin{array}{l}\text { 2MASS ID } \\
\text { Unit }\end{array}$ & Sp.T. & $\begin{array}{l}T_{\text {eff }} \\
(\mathrm{K})\end{array}$ & $\begin{array}{c}L_{\star} \\
\left(L_{\odot}\right)\end{array}$ & $\begin{array}{c}R_{\star} \\
\left(R_{\odot}\right)\end{array}$ & $\begin{array}{c}M_{\star} \\
\left(M_{\odot}\right)\end{array}$ & $\begin{array}{l}\text { err } \\
\left(M_{\odot}\right)\end{array}$ & $\begin{array}{l}L_{\mathrm{acc}} \\
\left(L_{\odot}\right)\end{array}$ & $\begin{array}{l}M_{\text {dust }} \\
\left(M_{\oplus}\right)\end{array}$ & $\begin{array}{c}\text { err } \\
\left(M_{\oplus}\right)\end{array}$ & $\begin{array}{l}\text { Detect } \\
(\mathrm{T} / \mathrm{F})\end{array}$ & $\begin{array}{c}\dot{M}_{\mathrm{acc}} \\
\left(M_{\odot} \mathrm{yr}^{-1}\right)\end{array}$ & $\begin{array}{c}\text { err } \\
\left(M_{\odot} \mathrm{yr}^{-1}\right)\end{array}$ & $\begin{array}{l}\text { Detect } \\
(\mathrm{T} / \mathrm{F})\end{array}$ & Region & Exclude \\
\hline J10533978-7712338 & M2 & 3560 & -1.80 & -0.48 & -0.41 & 0.11 & -4.56 & 0.19 & 0.0746 & True & -12.03 & 0.29 & True & Cha I & $\overline{\text { underlum }}$ \\
\hline J10555973-7724399 & K7 & 4060 & -0.74 & -0.07 & -0.13 & 0.05 & -1.25 & 1.06 & 0.0168 & True & -8.58 & 0.28 & True & Cha I & $\ldots$ \\
\hline $\mathrm{J} 10561638-7630530$ & M6.5 & 2935 & -1.10 & 0.04 & -0.96 & 0.07 & -4.55 & 0.12 & 0.0174 & True & -10.95 & 0.28 & False & Cha I & $\ldots$ \\
\hline J10563044-7711393 & K7 & 4060 & -0.37 & 0.12 & -0.07 & 0.17 & -2.24 & 1.59 & 0.0041 & True & -9.45 & 0.32 & True & Cha I & $\ldots$ \\
\hline J10574219-7659356 & M3 & 3415 & -0.28 & 0.32 & -0.52 & 0.05 & -1.98 & 0.48 & 0.0395 & True & -8.54 & 0.27 & True & Cha I & $\ldots$ \\
\hline
\end{tabular}

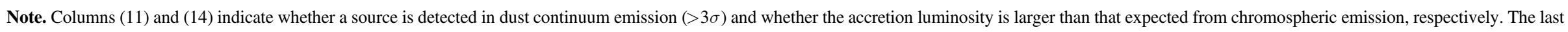
column denotes the reason why sources are not included.

(This table is available in its entirety in machine-readable form.) 


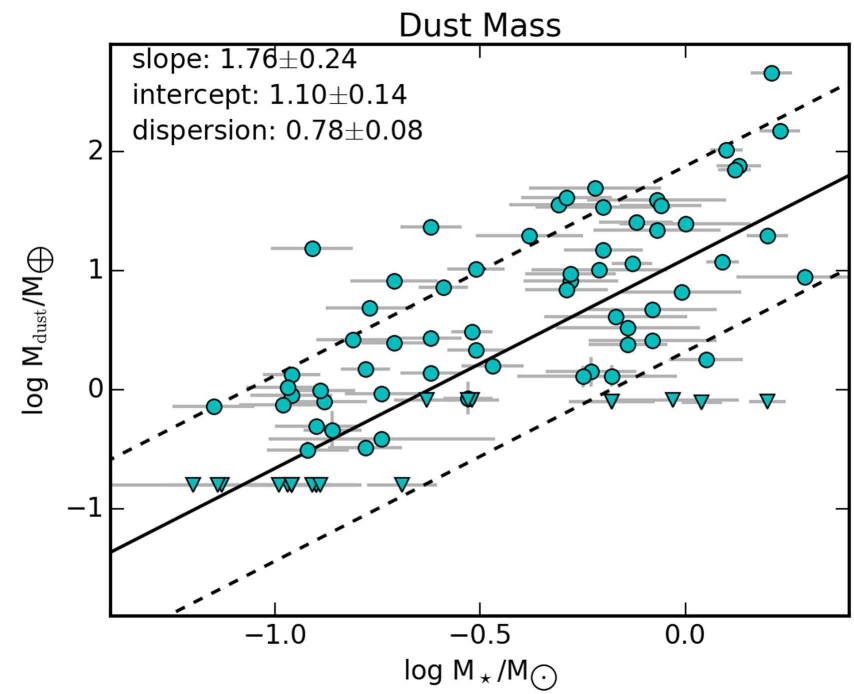

Figure 1. Dust mass $\left(T_{\text {dust }}=20 \mathrm{~K}\right)$ vs. stellar mass for sources in Chamaeleon I. Triangles denote $3 \sigma$ upper limits for sources that are not detected with ALMA. The solid line shows the best-fit regression including upper limits. The $1 \sigma$ dispersion around the best fit is indicated with dashed lines. The $M_{\text {dust }}-M_{\star}$ relation is steeper than linear, consistent with previous work.

We calculate the mass accretion rate, $\dot{M}_{\text {acc }}$, from the accretion luminosity, $L_{\text {acc }}$, following Hartmann et al. (1998):

$$
\dot{M}_{\mathrm{acc}}=1.25 \frac{L_{\mathrm{acc}} R_{\star}}{G M_{\star}},
$$

where $R_{\star}$ is the stellar radius, $M_{\star}$ is the stellar mass, and $G$ is the gravitational constant. The pre-factor 1.25 corresponds to a magnetospheric cavity size of 5 stellar radii, chosen to be consistent with Alcalá et al. (2017) and Manara et al. (2017). We propagate the errors on accretion luminosity ( 0.25 dex $)$ and stellar parameters to calculate the error on the mass accretion rate, which is typically 0.3 dex (see Table 1 ). The difference in the accretion rates compared to Manara et al. (2017) using the Siess et al. (2000) and Baraffe et al. (2015) evolutionary tracks is small, with a median deviation of $\sim 1 \%$ and a maximum of $6 \%$. The mass accretion rates as a function of stellar mass are shown in Figure 2.

\subsection{Lupus}

The data set of Lupus is based on the ALMA survey by Ansdell et al. (2016) and the X-Shooter survey by Alcalá et al. $(2014,2017)$. We follow the same procedure to derive a consistent data set as for Chamaeleon I. For consistency, we use the stellar masses and dust masses from Pascucci et al. (2016), which were derived using the same stellar evolutionary models and assumptions for the dust temperature and opacity as those for Chamaeleon I. We recalculate the mass accretion rate from the accretion luminosity using Equation (2). The difference in mass accretion rates with those derived using the Siess et al. (2000) and Baraffe et al. (2015) evolutionary tracks by Alcalá et al. (2017) are again small, with a median deviation of $\sim 4 \%$ and a maximum of $30 \%$.

\section{Analysis}

We first analyze the correlation between dust mass, stellar mass, and mass accretion rate for Chamaeleon I. In Section 3.2 we present a joint analysis including the Lupus data.

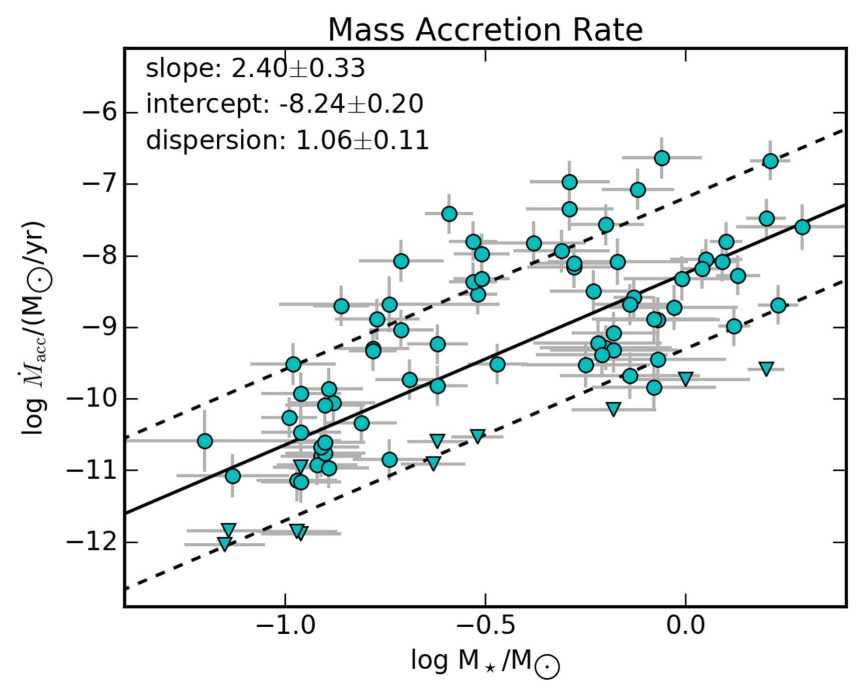

Figure 2. Mass accretion rates vs. stellar mass for sources in Chamaeleon I. Triangles denote dubious accretors for sources with an accretion luminosity consistent with chromospheric activity. The solid line shows the best-fit regression treating dubious accretors as upper limits. The $1 \sigma$ dispersion around the best fit is indicated with dashed lines. The $\dot{M}_{\text {acc }}-M_{\star}$ relation is quadratic, consistent with previous work.

Throughout this section, we use the Python version of linmix ${ }^{11}$ (Kelly 2007) for linear regression to estimate bestfit parameters for the mean slope and intercept, the intrinsic dispersion around the mean trend, and the correlation coefficient. Linmix takes into account measurement errors in both dimensions and upper limits (censored data) in one dimension.

\subsection{Chamaeleon I}

The dependences of dust mass and mass accretion rate on stellar mass have previously been derived by Pascucci et al. (2016) and Manara et al. (2017), respectively. We refit these correlations to verify that our sample selection and the use of different stellar evolutionary models from Manara et al. (2017) do not influence our results.

Figure 1 shows the measured dust masses, $M_{\text {dust }}$, as a function of stellar mass, $M_{\star}$. The best-fit relation between dust mass and stellar mass is described by

$$
\log \left(\frac{M_{\text {dust }}}{M_{\oplus}}\right)=1.8( \pm 0.2) \log \left(\frac{M_{\star}}{M_{\odot}}\right)+1.1( \pm 0.1)
$$

and shown as the solid line in Figure 1. The $1 \sigma$ dispersion is $0.8 \pm 0.1$ dex (dashed lines), and the correlation coefficient is $r=0.7 \pm 0.1$. These results are consistent with those in Pascucci et al. (2016) within the reported uncertainties. Figure 2 shows the stellar mass accretion rate, $\dot{M}_{\text {acc }}$, as a function of stellar mass, $M_{\star}$. The best-fit relation between mass accretion rate and stellar mass, treating dubious accretors as upper limits, is described by

$$
\log \left(\frac{\dot{M}_{\mathrm{acc}}}{M_{\odot} \mathrm{yr}^{-1}}\right)=2.4( \pm 0.3) \log \left(\frac{M_{\star}}{M_{\odot}}\right)-8.3( \pm 0.2),
$$

\footnotetext{
${ }^{11}$ https://github.com/jmeyers314/linmix
} 


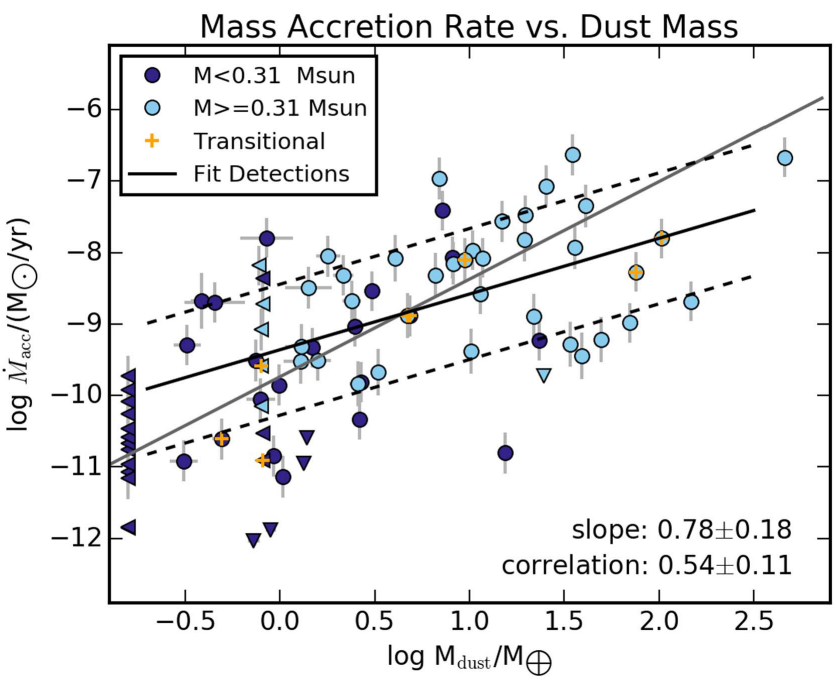

Figure 3. Dust masses $\left(T_{\text {dust }}=20 \mathrm{~K}\right)$ vs. mass accretion rates of sources in Chamaeleon I. Dubious accretors (left-facing triangles) and $3 \sigma$ nondetections of the dust (downward-facing triangles) are not included in estimating the bestfit $\dot{M}_{\text {acc }}-M_{\text {dust }}$ relation (back solid line) and intrinsic dispersion (dashed lines), which has a correlation coefficient of $r \approx 0.6$. Known transition disks are marked with yellow crosses and do not appear to be outliers. The sample is color-coded by stellar mass, with the low-mass half in purple and the high-mass half in light blue. The underlying distribution of stellar mass follows the expected correlation (gray solid line) based on the $M_{\text {dust }}-M_{\star}$ and $\dot{M}_{\text {acc }}-M_{\star}$ relations from Figures 1 and 2, respectively, with low (high) dust mass and low (high) mass accretion rates in the lower left (upper right) corner.

with a dispersion of $1.1 \pm 0.1$ dex and a correlation coefficient of $r=0.7 \pm 0.1$. These results are, within the uncertainties, consistent with the linear regression in Manara et al. (2017).

\subsubsection{Mass Accretion Rate versus Dust Mass}

We investigate the relation between the dust mass and mass accretion rates following the analysis in Manara et al. (2016b). Figure 3 shows the stellar mass accretion rate versus dust mass in Chamaeleon I. Known transition disks are marked in red, but they do not appear to be outliers in this distribution, motivating our choice in Section 2.1 to include them in the sample.

We find a moderate correlation between $M_{\text {dust }}$ and $\dot{M}_{\text {acc }}$, $r=0.6 \pm 0.1$, fitting only sources with detections in both dimensions. The best-fit relation is given by

$$
\log \left(\frac{\dot{M}_{\mathrm{acc}}}{M_{\odot} \mathrm{yr}^{-1}}\right)=0.8( \pm 0.2) \log \left(\frac{M_{\mathrm{dust}}}{M_{\oplus}}\right)-9.3( \pm 0.2),
$$

with a dispersion of $0.9 \pm 0.4$ dex. These values are consistent with those derived for Lupus (Manara et al. 2016b), except for the dispersion, which is significantly larger, owing to the larger dispersion in mass accretion rates in Chamaeleon I compared to Lupus (Manara et al. 2017). The slope is within errors consistent with a linear correlation between dust mass and mass accretion rate.

The slope is flatter than the expected correlation based on Equations (3) and (4) (solid gray line; $\log \dot{M}_{\text {acc }} \propto 1.4 \pm$ $\left.0.3 \log M_{\text {dust }}\right)$. A concern could be that the flatter slope may have been introduced by omitting upper limits in the fitting procedure. Because linmix does not support upper limits in two dimensions simultaneously, we separately fit the upper limits in each dimension. Including upper limits on $\dot{M}_{\text {acc }}$ yields a linear slope of $1.0 \pm 0.2$, while including upper limits on $M_{\text {dust }}$ yields a steeper-than-linear slope of $1.6 \pm 0.2$. The latter is more consistent with the $\dot{M}_{\text {acc }}-M_{\text {dust }}$ correlation based on the stellar-mass dependencies of both parameters (Equations (3) and (4)). Given the uncertainties in the derived values, we conclude that there is no clear evidence that the $\dot{M}_{\text {acc }}-M_{\text {dust }}$ relation deviates from a linear trend.

\subsubsection{Characterizing the Observed Scatter}

A linear correlation between dust mass and mass accretion rate is consistent with the idea that protoplanetary disks evolve viscously (e.g., Manara et al. 2016b, and references therein). There is, however, significant scatter around the best-fit $M_{\text {dust }}-\dot{M}_{\text {acc }}$ relation not predicted by constant- $\alpha$ disk models (e.g., Dullemond et al. 2006). To characterize the intrinsic scatter in observed dust masses and mass accretion rates, we divide out the fitted trend with stellar mass from the dust mass and mass accretion rate. We define two new quantities, $\Delta M_{\text {dust }}$ and $\Delta \dot{M}_{\text {acc }}$, that represent how much a given source deviates from the best-fit value at its stellar mass. The stellar-massdetrended dust mass, $\Delta M_{\text {dust }}$, is given by

$$
\Delta M_{\mathrm{dust}}=\log \left(\frac{M_{\mathrm{dust}}}{M_{\oplus}}\right)-\left(A_{d} \log \left(\frac{M_{\star}}{M_{\odot}}\right)+B_{d}\right),
$$

where the coefficients $A_{d}=1.77$ and $B_{d}=12.6$ are taken from Equation (3). Positive values of $\Delta M_{\text {dust }}$ indicate a higher-thanaverage dust mass at that stellar mass, i.e., the source is above the mean trend in Figure 1 (solid line). Negative values of $\Delta M_{\text {dust }}$ indicate a lower-than-average dust mass, and the source is located below the best-fit trend in Figure 1.

The stellar-mass-detrended mass accretion rate, $\Delta \dot{M}_{\mathrm{acc}}$, is given by

$$
\Delta \dot{M}_{\mathrm{acc}}=\log \left(\frac{\dot{M}_{\mathrm{acc}}}{M_{\odot} \mathrm{yr}^{-1}}\right)-\left(A_{a} \log \left(\frac{M_{\star}}{M_{\odot}}\right)+B_{a}\right),
$$

where the coefficients $A_{a}=2.4$ and $B_{d}=5.5 \times 10^{-9}$ are taken from Equation (4). The stellar-mass-detrended values for the dust mass $\left(\Delta M_{\text {dust }}\right)$ and mass accretion rate $\left(\Delta \dot{M}_{\text {acc }}\right)$ are shown in Figure 4.

There is no clear trend visible between the detrended quantities $\Delta M_{\text {dust }}$ and $\Delta \dot{M}_{\text {acc }}$, in contrast to the $\dot{M}_{\text {acc }}-M_{\text {dust }}$ plot. The lower-mass half (purple) and higher-mass half (cyan) of the sample show a similar spatial distribution, indicating no residual trend with stellar mass. Fitting detections only, a weak $(r=0.28 \pm 0.15)$ correlation may be present, with a slope of $0.36 \pm 0.14$ that deviates significantly from a linear correlation. Including upper limits on the mass accretion rate or dust mass yields a weaker $(r=0.19 \pm 0.14)$ or stronger correlation $(r=0.35 \pm 0.12)$, respectively, with similar slopes. Treating dubious accretors as detections does not significantly affect these results. In all cases, a strong correlation as may be expected from constant- $\alpha$ disk models is not present. We test for the robustness of this result by increasing the sample size in Section 3.2.

\subsection{Chamaeleon I and Lupus Combined}

The dust masses, mass accretion rates, and stellar masses of the combined Lupus/Chamaeleon I data set are shown in 


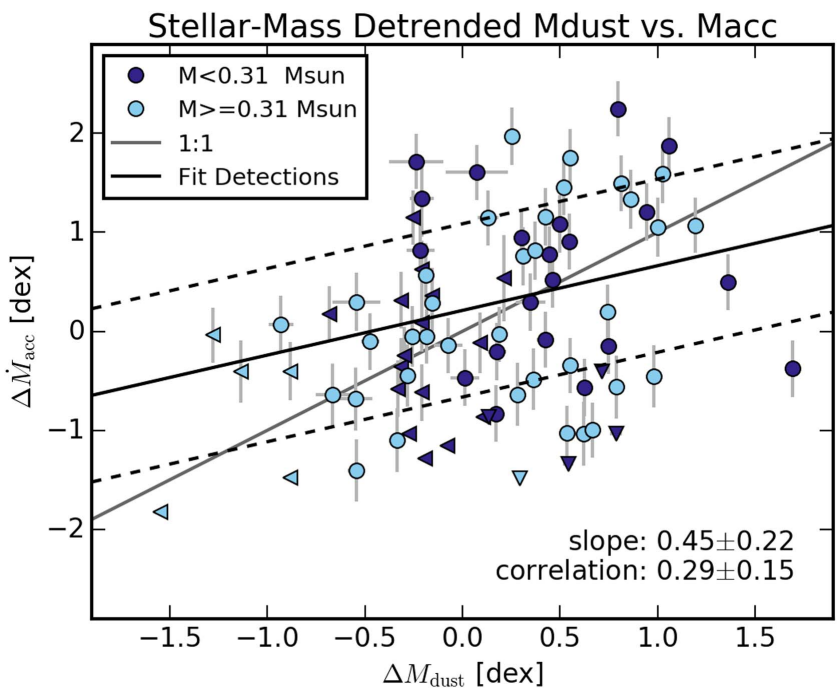

Figure 4. Stellar-mass-detrended mass accretion rate, $\Delta \dot{M}_{\mathrm{acc}}$, vs. dust mass, $\Delta M_{\text {dust }}$. The gray line shows the linear correlation expected from disk models with a constant $\alpha$, which is not recovered by linear regression. There is no clear separation between the lower stellar mass (purple) and higher stellar mass (light blue) half of the sample. Black lines show the best-fit regression curve (solid line) and $1 \sigma$ dispersion (dashed line). A correlation-if present-is weak $(r \approx 0.2-0.4$, depending on how upper limits and dubious accretors are treated; see text). The lack of a clear correlation indicates that for stars of comparable mass, the mass accretion rate does not depend on dust mass.

Figure 5, with a figure layout equivalent to Figures 1-4. The dust disk masses of both regions show a similar dependence on stellar mass, but one that is different from the older Upper Sco star-forming region (Pascucci et al. 2016). The mass accretion rates show a similar mean trend to stellar mass (top right panel; see also Alcalá et al. 2017; Manara et al. 2017), though the dispersion around the mean trend in Lupus is smaller (Alcalá et al. 2014, 2017). As discussed in Section 3.1.1, the mass accretion rates show the same correlation with dust mass in Chamaeleon I as in Lupus.

We recover the $\dot{M}_{\text {acc }}-M_{\text {dust }}$ correlation in the combined Lupus-Chamaeleon I data set with a correlation coefficient of $0.55 \pm 0.08$, a slope of $0.75 \pm 0.12$, and a dispersion of $0.80 \pm 0.07$ (bottom left panel of Figure 5). These results are consistent with estimates for the individual star-forming regions, but derived at higher statistical confidence due to the larger sample size. We show in Appendix A that considering a limited stellar mass range does not lead to a stronger correlation. We investigate a possible underlying correlation with stellar mass by detrending $M_{\text {dust }}$ and $\dot{M}_{\text {acc }}$ with the same procedure as described in Section 3.1.2. The stellar-massdetrended dust mass, $\Delta M_{\text {dust }}$, is calculated from Equation (3) with coefficients $A_{d}=1.75$ and $B_{d}=10^{1.3}$ derived from fitting the combined data set (solid line in top left panel). The stellarmass-detrended mass accretion rate, $\Delta \dot{M}_{\mathrm{acc}}$, is derived using Equation (4) with coefficients $A_{a}=2.1$ and $B_{a}=10^{-8.2}$ derived from fitting the combined data set (solid line in top right panel).

We again find a weak correlation $(r=0.27 \pm 0.10)$ between $\Delta M_{\text {dust }}$ and $\Delta \dot{M}_{\text {acc }}$, with a slope of $0.36 \pm 0.14$ that is inconsistent with linear. The inclusion of upper limits in either dimension and treating dubious accretors as upper limits do not significantly change the strength of the correlation.

\section{Simulations}

In this section we make a quantitative comparison between disk evolutionary models and the observed relations between dust mass, mass accretion rate, and stellar mass. We take a forward-modeling approach, simulating an ensemble of evolving disks and generating synthetic observations that are analyzed with the same statistical tools and procedures as the observations.

We use the Chambers (2009) analytic disk model to simulate the time evolution of a protoplanetary disk. This model calculates the surface-density evolution of an irradiated disk due to a (turbulent) viscosity, parameterized by the dimensionless quantity $\alpha$ (Shakura \& Sunyaev 1973; see Equation (1) and introduction), which is kept constant throughout the disk and over time. $^{12}$

The disk evolution depends on a number of initial parameters. The stellar radius, $R_{\star}$, and luminosity, $L_{\star}$, are calculated from the stellar mass, $M_{\star}$, using the combined (nonmagnetic) Baraffe et al. (2015) and Feiden (2016) evolutionary tracks as in Pascucci et al. (2016). The initial disk mass $\left(M_{\text {disk, } 0}\right)$, radius $\left(R_{\text {out }, 0}\right)$, opacity, and viscosity are adopted from the first example in Chambers (2009), except for the last model, which explores nonstandard initial conditions (see Section 4.3). Each disk is evolved until the age of the system, $t_{\text {disk }}$. A stellar-mass dependency of $M_{\star}^{1.9}$ is introduced to the initial disk mass to fit the observed scalings between $M_{\text {dust }}-M_{\star}$. Because in a constant $\alpha$ disk the dust mass and mass accretion rate are coupled, this also introduces a stellar-mass dependency in the resulting $\dot{M}_{\mathrm{acc}}-M_{\star}$ relation.

To match the observed scatter in $M_{\text {dust }}$ and $\dot{M}_{\text {acc }}$, we introduce a dispersion in disk model parameters $\left(M_{\text {disk }, 0}\right.$, $R_{\text {out }, 0}, t_{\text {disk }}, \alpha$ ). The dispersions in initial disk mass and radius represent variations in disk initial conditions. The dispersion in disk lifetime represents an age spread in the cloud. The dispersion in viscosity parameter $\alpha$ represents variations in angular momentum transport efficiency between disks. We also introduce two additional parameters that can contribute to the observed scatter. The gas-to-dust ratio, $f_{\text {gtd }}=M_{\text {gas }} / M_{\text {dust }}$, allows us to convert the modeled gas disk mass to a dust mass. The dispersion in $f_{\mathrm{gtd}}$ reflects both physical processes that may alter the gas-to-dust ratio (see Section 5.4), as well as uncertainties in deriving the dust mass from the unresolved millimeter flux (see Section 5.1). The other parameter, $f_{\text {acc }}=\dot{M}_{\text {acc }} / \dot{M}_{\text {disk }}$, represents accretion rate variability and is defined as the ratio of the observed instantaneous stellar mass accretion rate to the time-averaged mass accretion rate of the disk.

We simulate a survey similar in size to the combined Lupus and Chamaeleon I sample, with 140 stars randomly drawn between 0.1 and $1.6 M_{\odot}$ from a lognormal initial mass function (Chabrier 2003). For each star we run the Chambers (2009) disk model with free parameters $\left(M_{\text {disk }, 0}, R_{\text {out }, 0}, t_{\text {disk }}, \alpha, f_{\text {gtd }}\right.$, and $f_{\text {acc }}$ ) randomly sampled from a lognormal distribution, with mean and standard deviation as in Table 2. The gas-to-dust ratio $\left(f_{\text {gtd }}\right)$ was increased to 300 to fit the intercept of both $M_{\text {dust }}$ and $\dot{M}_{\text {acc }}$. The choice of gas-to-dust ratio is not unique, and a ratio of 100 can be achieved by a different conversion of millimeter flux to dust mass, by either lowering the dust opacity or decreasing the dust temperature. These values reproduce the

\footnotetext{
12 Note that, unlike in self-similar solutions, viscosity changes over time and it is not restricted to a radial power-law dependence.
} 

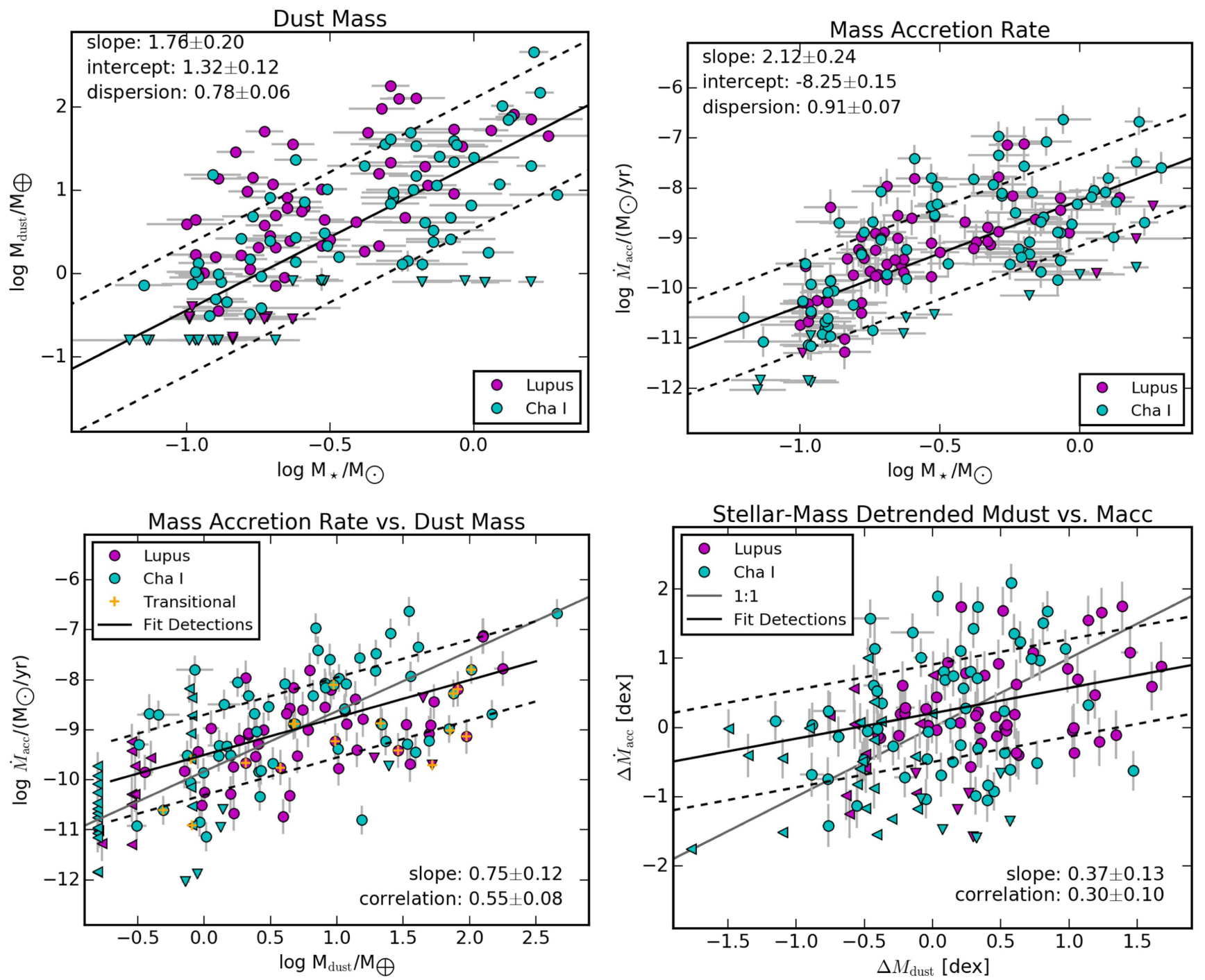

Figure 5. Dust masses, mass accretion rates, and stellar masses for the combined Chamaeleon I (cyan) and Lupus (pink) samples. Top left: dust mass vs. stellar mass, as in Figure 1. Top right: mass accretion rate vs. stellar mass, as in Figure 2. Bottom left: dust mass vs. mass accretion rate, as in Figure 3, but color-coded by starforming region. Bottom right: detrended dust mass vs. mass accretion, as in Figure 4, but again color-coded by star-forming region.

best-fit $M_{\text {dust }}-M_{\star}$ and $\dot{M}_{\text {acc }}-M_{\star}$ relations for the combined Lupus-Chamaeleon I data set (e.g., Figure 5).

Synthetic observations are conducted using a Monte Carlo simulation by perturbing each observable $\left(M_{\star}, M_{\text {dust }}, \dot{M}_{\text {acc }}\right)$ with an observational uncertainty of $0.1,0.1$, and $0.25 \mathrm{dex}$, respectively. The simulated observables are considered upper limits (or dubious accretors) if the dust mass (or mass accretion rate) falls below the detection threshold of the survey (see Pascucci et al. 2016; Manara et al. 2017, for details).

\subsection{Model vis1}

First, we simulate a disk model where the observed scatter in $M_{\text {dust }}$ and $\dot{M}_{\text {acc }}$ arises from a dispersion in initial disk conditions as in Hartmann et al. (1998) and Dullemond et al. (2006). The dust mass is assumed to be a direct tracer of the gas mass (no dispersion in $f_{\text {gtd }}$ ), and the instantaneous mass accretion rate is a direct tracers of the time-averaged mass accretion rate (no dispersion in $f_{\text {acc }}$ ). The stellar-mass dependencies in dust mass and mass accretion rate are, within their uncertainties, consistent with the observed values (Figure 6). Disk-to-disk variations in the initial disk mass, radius, $\alpha$, and age create a scatter of $\sim 0.8$ dex around the best-fit $M_{\text {dust }}-M_{\star}$ and $\dot{M}_{\text {acc }}-M_{\star}$ relations.

The vis 1 set of disk models has a median viscous timescale of $0.1 \mathrm{Myr}$, significantly shorter than the disk lifetime, and produces a nearly linear $M_{\text {dust }}-\dot{M}_{\text {acc }}$ relation. Taking into account measurement errors and upper limits, the correlation is recovered at high confidence with negligible scatter. The correlation is much stronger than observed, with a correlation coefficient of unity compared to $r=0.6$ for the Lupus-Chamaeleon I data.

We use Equations (6) and (7) to detrend the synthetic observations and calculate $\Delta M_{\text {dust }}$ and $\Delta \dot{M}_{\text {acc }}$, where the coefficients $A_{D}=1.4$ and $B_{D}=8.5$ are fitted to synthetic $M_{\text {dust }}-M_{\star}$ observations and $A_{A}=2.0$ and $A_{D}=5 \times 10^{-8}$ are fitted to synthetic $\dot{M}_{\text {acc }}-M_{\star}$ observations. The strong linear correlation between $\Delta M_{\text {dust }}$ and $\Delta \dot{M}_{\text {acc }}$ remains present in the simulated data after detrending.

The vis 1 model presented here is not a unique solution. A degenerate set of parameters exist where the dispersion in initial disk mass can be traded off for higher dispersions in disk outer radius, lifetime, and/or viscosity, and vice versa, without affecting the strength of the observed correlations. The 
Table 2

Initial Conditions for the Simulated Disk Models

\begin{tabular}{|c|c|c|c|c|c|c|c|c|c|c|c|c|c|}
\hline Model & & $M_{\text {disk }, 0}\left(M_{\odot}\right)$ & & $R_{\text {out }}$ & & & & $\alpha$ & & $f_{\text {gtd }}$ & & $f_{\text {acc }}$ & \\
\hline \multirow[t]{2}{*}{ vis1 } & Mean & $0.1 M_{\star}^{1.9}$ & & 33 & & 2 & & 0.01 & & 300 & & 1 & \\
\hline & Disp. (dex) & & 0.8 & & 0.3 & & 0.3 & & 0.3 & & 0 & & 0 \\
\hline \multirow[t]{2}{*}{ vis2 } & Mean & $0.1 M_{\star}^{1.9}$ & & 33 & & 2 & & 0.01 & & 300 & & 1 & \\
\hline & Disp. (dex) & & 0.3 & & 0.3 & & 0.3 & & 0.3 & & 0.4 & & 0.7 \\
\hline \multirow[t]{2}{*}{ vis3 } & Mean & $0.4 M_{\star}^{1.9}$ & & 33 & & 2 & & 0.001 & & 1000 & & 1 & \\
\hline & Disp. (dex) & & 0.5 & & 0.5 & & 0.5 & & 2.0 & & 0 & & 0 \\
\hline
\end{tabular}

Note. Parameters for each disk are randomly drawn from a lognormal distribution with the listed mean and standard deviation (dispersion). $f_{\text {gtd }}$ is the gas-to-dust ratio in the disk at time of observation. $f_{\text {acc }}$ is the ratio of the measured mass accretion rate to the time-averaged mass accretion rate, representing accretion variability.

observable dispersion is less sensitive to these parameters than to the initial disk mass. For example, a dispersion in the outer disk radii of 0.8 dex results in a scatter in the observed dust mass and mass accretion rates of $\sim 0.3-0.4 \mathrm{dex}$. A dispersion in the disk mass of $0.5 \mathrm{dex}$ was independently derived by Armitage et al. (2003) by modeling the fraction of stars with disks as a function of time. We explored a large range of initial conditions (a factor of 10 in initial disk mass and outer radius, and a factor of 100 in $\alpha$ ) and consistently find that these solutions produce strong correlations between $M_{\text {dust }}$ and $\dot{M}_{\text {acc }}$ $(r>0.95)$ and between $\Delta M_{\text {dust }}$ and $\Delta \dot{M}_{\text {acc }}(r>0.9)$, except when long viscous timescales are used in combination with nonstandard input parameters (see Section 4.3, vis3 model). The strong correlations show that the zeroth-order assumptions of dust mass and mass accretion rate as direct tracers of disk evolution are inconsistent with the observed moderate and weak correlations within the framework of a viscously evolved constant- $\alpha$ disk model.

\subsection{Model vis2}

The observed dust mass and mass accretion rate may not be perfect tracers of the disk conditions. Spatial and temporal variations in disk viscosity, as well as the accretion process near the stellar magnetosphere, lead to accretion rate variability. At the same time, variations from disk to disk in dust temperature, opacity, and gas-to-dust mass ratio may also contribute to the observed scatter in millimeter fluxes and hence dust masses.

Here, we explore how large the influence of these two processes needs to be for the constant- $\alpha$ disk model to produce the observed scatter in the $M_{\text {dust }}-\dot{M}_{\text {acc }}$ relation, modeled by parameters $f_{\text {acc }}$ and $f_{\text {otd }}$. In model vis2 we reduce the dispersion in the initial disk mass to 0.3 dex, reducing the scatter in $M_{\text {dust }}$ and $\dot{M}_{\text {acc }}$. We add scatter to the observable mass accretion rate by introducing variability in the accretion rate, $f_{\text {acc }}$, of 0.7 dex. Similarly, we increase the scatter in the observable dust masses by adding disk-to-disk variations in the gas-to-dust ratio, $f_{\text {gtd }}$, of 0.7 dex.

This model provides a good fit to observed scatter in $M_{\text {dust }}$ and $\dot{M}_{\text {acc }}$ (Figure 7). In addition, the uncorrelated scatter weakens the observed relation between dust mass and mass accretion rate, and these parameters provide a good fit to the observed correlation between $M_{\text {dust }}$ and $\dot{M}_{\text {acc }}(r=0.6)$ and the stellar-mass-detrended $\Delta M_{\text {dust }}-\Delta \dot{M}_{\text {acc }}$ correlation $(r=0.3)$. We varied $f_{\text {acc }}$ and $f_{\text {gtd }}$ independently and found that both parameters need to be nonzero to explain the observed scatter in $M_{\text {dust }}$ and $\dot{M}_{\text {acc }}$. Although the intrinsic relation between $M_{\text {dust }}$ and $\dot{M}_{\text {acc }}$ in this model is linear, after applying upper limits the $M_{\text {dust }}-\dot{M}_{\text {acc }}$ relation recovered with linmix is shallower than linear, in agreement with the analysis of the observed values.

This model implies that (spatially unresolved) measurements of the dust mass and (instantaneous) measurements of mass accretion rates for individual objects may not be good tracers of the disk gas. In fact, the stellar mass is a more accurate predictor of disk mass $(\sigma=0.4 \mathrm{dex})$ compared to the measured dust mass $(\sigma=0.7 \mathrm{dex})$.

\subsection{Model vis3}

Lodato et al. (2017) have recently shown that, in the framework of self-similar solutions for viscous disks, one can reproduce the shallower-than-linear $M_{\mathrm{dust}}-\dot{M}_{\text {acc }}$ relation and the large scatter around it if most disks have not substantially evolved. Inspired by this work, we explore long viscous timescales in the context of the constant- $\alpha$ disk model and determine for which input parameters and spread we can reproduce the slope and scatter in the $M_{\text {dust }}-\dot{M}_{\text {acc }}$ and the stellar-mass-detrended $\Delta M_{\text {dust }}-\Delta \dot{M}_{\text {acc }}$ relation.

We find that the initial viscous timescale, $t_{\text {vis }, 0} \propto R_{\text {out }, 0}^{2} / \nu$, can be increased from the $\sim 0.1 \mathrm{Myr}$ in models visi and vis2 to $\sim 1$ Myr by decreasing the viscosity to $\alpha=0.001$ (or increasing the initial disk radius). Because the longer viscous timescale also reduces the mass accretion rate for a given disk mass, we increase the gas-to-dust ratio in model vis 3 by a factor of 3 to reproduce the observed values (we discuss the implications of these choices in Section 5). The correlation between $M_{\text {dust }}$ and $\dot{M}_{\text {acc }}$ weakens in combination with a large dispersion in the parameters that affect most the viscous time $\left(R_{\text {out }, 0}, \alpha\right)$. For instance, a model with a dispersion in $\alpha$ of $2 \mathrm{dex}$ and outer radius of $0.5 \mathrm{dex}$ can reproduce the observed relations and scatter around them (see vis 3 in Table 2 and Figure 8).

\section{Discussion}

The modeling carried out in the previous sections points out two possible solutions to the shallower-than-linear $M_{\text {dust }}-\dot{M}_{\text {acc }}$ relation and the large scatter between these quantities. The first possibility (vis2) is that (spatially unresolved) measurements of the dust mass and (instantaneous) measurements of mass accretion rates for individual objects are not good tracers of the disk gas in protoplanetary disks. In Section 5.1 we summarize the current status on these observables and discuss ways to reduce their uncertainties. The second possibility (vis 3 ) is that most $\sim 2-3 \mathrm{Myr}$ old disks have not viscously evolved 

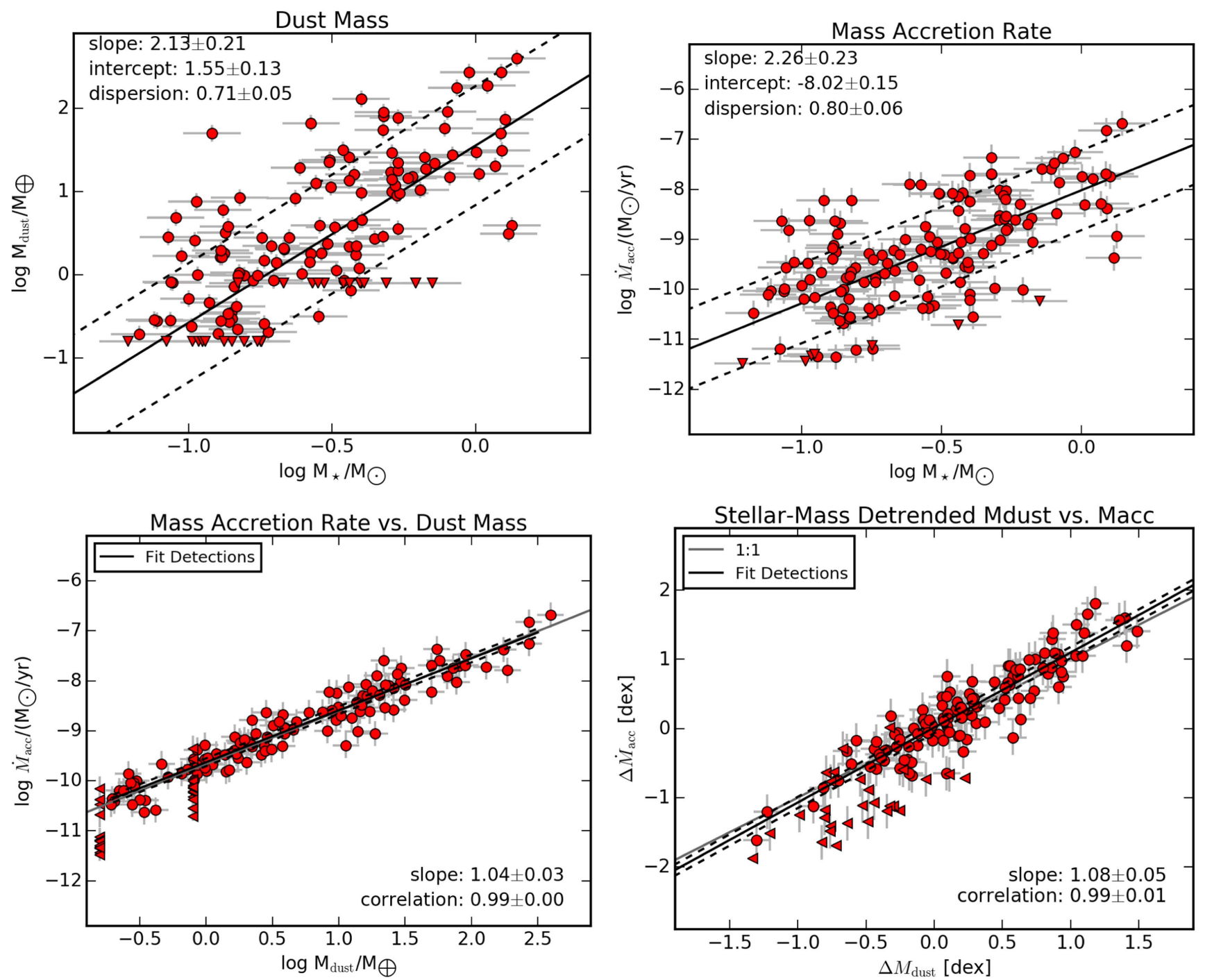

Figure 6. Synthetic observations for the disk model vis1. The panel layout is the same as for the observed Lupus-Chamaeleon I data set in Figure 5. The inferred correlations in the bottom two panels are much stronger than observed, indicating that a dispersion in initial disk mass is not the main factor determining the dispersion in dust mass and mass accretion rates.

substantially, and hence their birth properties (and scatter) remain imprinted in the observed $M_{\text {dust }}-\dot{M}_{\text {acc }}$ relation. We discuss in Section 5.2 the implications of this scenario and which observables are needed to test it. Finally, the constant- $\alpha$ disk model may not provide a good description of disk viscosity, and we discuss various other physical processes that could contribute to the observed scatter in the $M_{\text {dust }}-\dot{M}_{\text {acc }}$ relation (Section 5.4), as well as a completely different scenario based on (MHD) disk winds (Section 5.3).

\subsection{Mass Accretion Rates and Disk Masses: Current Status and Possible Improvements}

The mass accretion rates used in this paper are based on single-epoch observations. Accretion rate variability on different timescales will contribute to the observed scatter, as modeled through the dispersion in parameter $f_{\text {acc }}$. On timescales up to a year, rotational modulation of the accretion flow by the star introduces a variability of $\sim 0.4 \mathrm{dex}$ (Costigan et al. 2012; Venuti et al. 2015). We show in Appendix B that this short-term variability is not sufficient to explain the observed scatter, as variability would need to be of order
0.7 dex. Other multi-epoch studies also found that accretion rate variability is smaller than the observed scatter in mass accretion rates (e.g., Nguyen et al. 2009; Fang et al. 2013).

Constraining accretion variability on timescales longer than a year is challenging. FU Orionis objects undergo brightening events associated with large increases in mass accretion rate, though their duty cycle is unknown and they are primarily associated with young massive disks. EXORs undergo similar brightening events but at shorter timescales. Large accretion rate variations have been reported on long timescales, for example, the mass accretion rate of the Herbig Ae star HD 163296 has increased by $1.0 \mathrm{dex}$ in $\sim 15$ yr (Mendigutía et al. 2013). If such variations on decade-long timescales are common for $\mathrm{T}$ Tauri stars, repeated observations of accreting sources may provide a more accurate estimate of the timeaverage mass accretion rate. If variations in the accretion flow take place on timescales beyond that of modern astronomy $\left(10^{2}-10^{5} \mathrm{yr}\right)$, this may not be feasible.

The (dust) disk mass estimates from ALMA are calculated from the $887 \mu \mathrm{m}$ continuum flux assuming the same dust temperature, opacity, and gas-to-dust ratio for all disks. If these quantities vary from disk to disk, they may contribute to the 

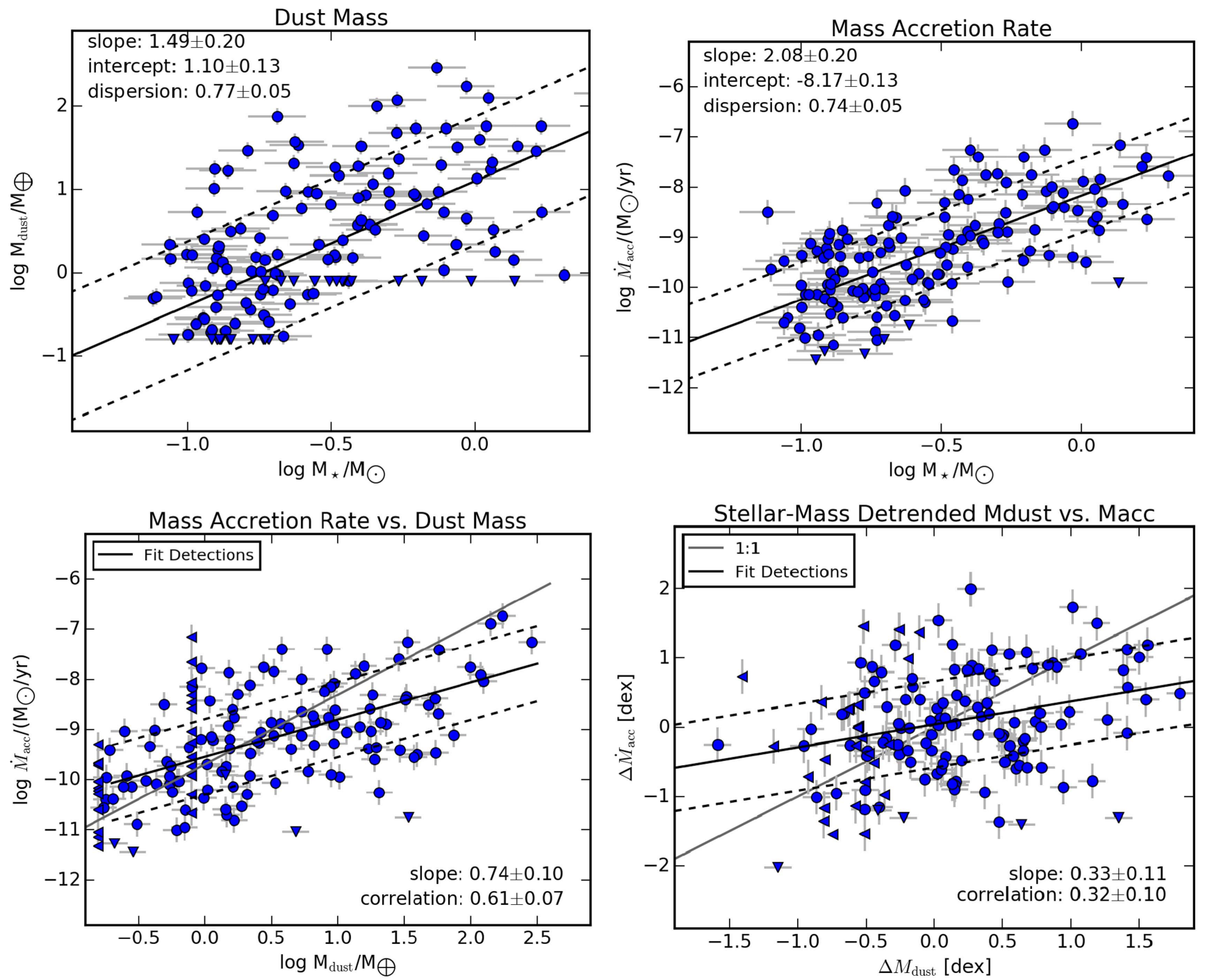

Figure 7. Synthetic observations for the disk model vis2. Same panels as in Figure 6.

observed scatter in millimeter fluxes, and hence disk masses, in the following way:

1. Disk Size. The characteristic temperature at which the disk emits depends on the spatial distribution of dust, in particular disk size (e.g., Hendler et al. 2017). Spatially resolved millimeter observations show that protoplanetary disks vary in size by an order of magnitude (e.g., Andrews et al. 2010, $\sigma \approx 0.4$ dex). In the optically thin limit $\left(T \propto R_{\text {disk }}^{-1 / 2}\right)$ these disk size variations would amount to a dispersion in millimeter fluxes of $\approx 0.2 \mathrm{dex}$, significantly smaller than the required dispersion in $f_{\mathrm{gtd}}$ of 0.7 dex.

2. Dust Opacity. The dust opacity at millimeter wavelengths depends on the grain size and composition (e.g., Draine 2006). Multiwavelength radio observations indicate that there are variations between protoplanetary disks in the spectral indices, indicative of different grain size distributions (e.g., Ricci et al. 2010). These variations in grain size distributions may correspond to variations in the dust opacity by an order of magnitude and may contribute significantly to the scatter in the observed millimeter fluxes. A better characterization of the grain size distributions using multiwavelength observations may therefore provide a more accurate estimate of the dust disk mass.

3. Disk Substructure. High spatial resolution spatial observations indicate substructure in some protoplanetary disks that is indicative of radial drift and particle trapping (e.g., van der Marel et al. 2013). Particle traps may be crucial in retaining a detectable amount of dust in the outer disk (e.g., Pinilla et al. 2012), and the location and strength of these traps may affect dust mass estimates based on spatially unresolved observations. However, the number of spatially resolved disks is currently not large enough to asses the relevance of particle traps on the millimeter flux.

Observations of the dust continuum at high spatial resolution and multiple wavelengths for a significant number of disks may be used to provide more accurate estimates of the (dust) disk mass. Using the vis 2 model, we predict that a reduction in the derived uncertainty on disk mass from 0.7 to 0.3 dex should produce a detectable correlation with $r>0.5$ if disks evolve like constant- $\alpha$ disks on short timescales ( $\ll 1 \mathrm{Myr})$. This corresponds to a scatter around the best-fit $M_{\text {dust }}-M_{\star}$ relation of 0.4 dex, versus 0.8 dex currently. Direct estimates of the gas mass for a large number of protoplanetary disks would be certainly preferable to test the $M_{\mathrm{disk}}-\dot{M}_{\mathrm{acc}}$ relation. 

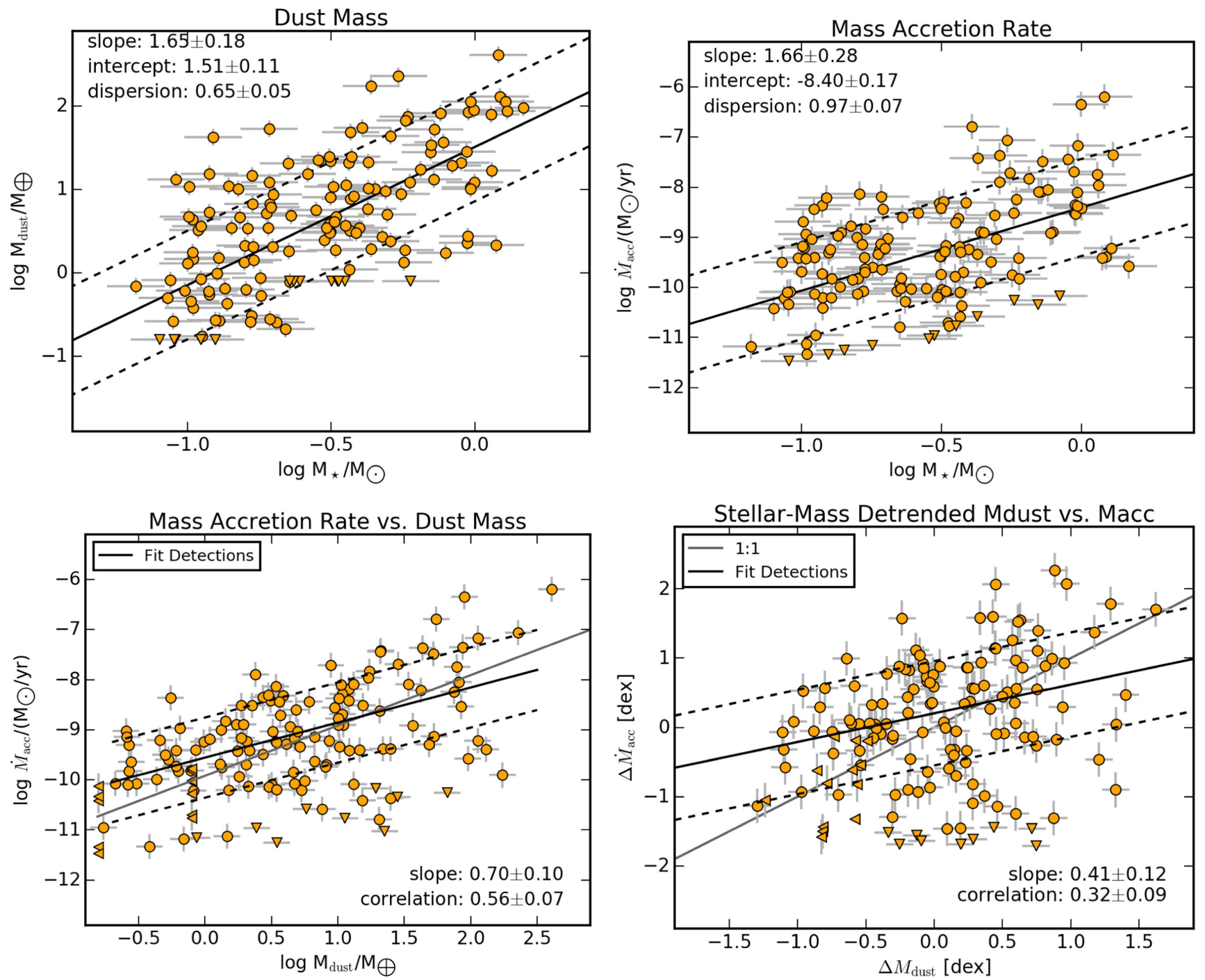

Figure 8. Synthetic observations for the disk model with a long viscous timescale, vis 3 . Same panels as in Figure 6.

\subsection{Slow Viscous Evolution}

Lodato et al. (2017) suggested a scenario according to which most $\sim 2-3$ Myr old disks have not yet substantially evolved, and the viscosity has a steeper radial dependence than an irradiated disk where $\alpha$ is a constant. In the framework of the constant- $\alpha$ disk model vis 3 this requires a low viscosity (or large radii at birth) in combination with a higher gas mass to enable accretion onto the star at the observed rates, since $M_{\text {disk }} \propto t_{\mathrm{vis}} \dot{M}_{\mathrm{acc}}$. An implication of this model is that dust masses of protoplanetary disks are systematically underestimated by a factor of $\sim 3-10$. While possible, the absolute value of the dust opacity is largely unknown (e.g., Beckwith et al. 2000); this seems unlikely as it would imply that a significant fraction of the 2-3 Myr old disks in Lupus and Chamaeleon I are gravitationally unstable (see, e.g., Figure 6 in Pascucci et al. 2016). Although these ALMA surveys are rather shallow, none of the Lupus-Chamaeleon I disks, even the brightest and presumably most massive ones, show the spiral structures that develop in gravitationally unstable disks (e.g., Kratter \& Lodato 2016; Rice 2016).

Disks disperse on timescales similar to the age of Chamaeleon I and Lupus, as evident from the decrease in the fraction of stars with a disk and with detectable accretion as cluster age increases (e.g., Mamajek 2009; Fedele et al. 2010). In the standard viscous evolution scenario, disks accrete most of their mass until star-driven photoevaporation takes over and quickly disperses the disk, the two-timescale disk dispersal (e.g., Ercolano \& Pascucci 2017). Even with X-ray- and far-UV-driven photoevaporation, current models estimate that the total mass lost to photoevaporation amounts to only $\sim 20 \%-30 \%$ of the initial disk mass (see Figure 4 in Alexander et al. 2014). In the slow viscous evolution scenario, disks do not lose a significant fraction of their initial mass through accretion on million-year timescales; hence, even more efficient photoevaporation or a different mechanism would be required to disperse them.

The evolution of mass accretion rates could provide important constraints to the slow viscous evolution scenario. Accretion rates in the Class I stage are on average higher than those in the Class II stage, $\sim 10^{-7} M_{\odot} \mathrm{yr}^{-1}$ versus $\sim 10^{-8} M_{\odot} \mathrm{yr}^{-1} \quad$ (White \& Hillenbrand 2004; Nisini et al. 2005; Caratti et al. 2012). In addition, mass accretion rates of Class II sources appear to decrease on a million-year timescale (Hartmann et al. 1998; Sicilia-Aguilar et al. 2010; Antoniucci et al. 2014; Hartmann et al. 2016), consistent with the standard viscous evolution scenario, but the spread is large 

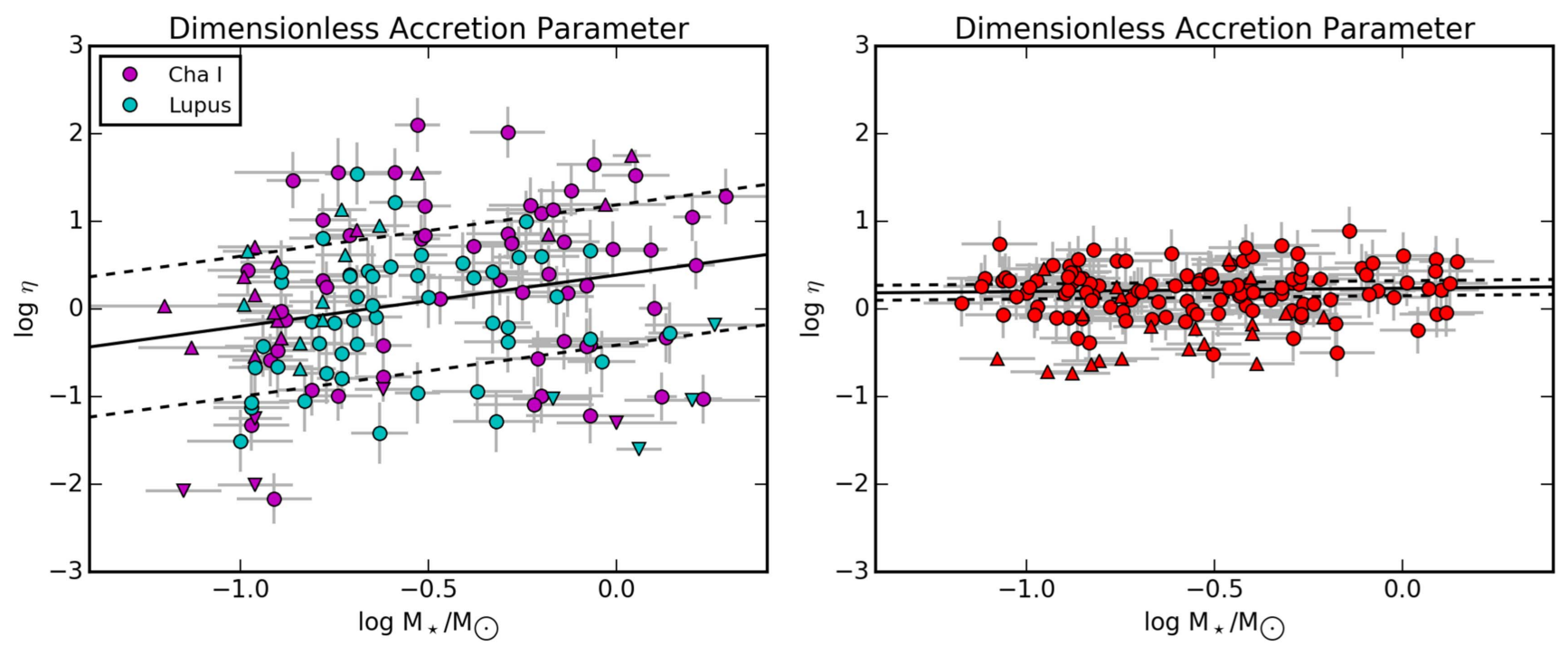

Figure 9. Dimensionless accretion parameter $\eta=\dot{M}_{\text {acc }} t_{\text {disk }} /\left(100 M_{\text {dust }}\right)$ for observed disks in Lupus and Chamaeleon I (left) and for disk model vis 1 (right).

to be certain. Mass accretion rate measurements for older regions, e.g., the $\sim 5-10 \mathrm{Myr}$ old Upper Sco, would be extremely valuable, as the scatter around the $M_{\text {dust }}-\dot{M}_{\text {acc }}$ relation will be tighter. Evolving model vis3 to $7 \mathrm{Myr}$ indicates that a correlation between $\Delta M_{\text {dust }}$ and $\Delta \dot{M}_{\text {acc }}$ may become detectable at a $>3 \sigma$ level even for the long viscous time.

Finally, estimates of gas disk radii would also be important to test disk evolutionary models. In the context of viscous disk models, gas disks spread with time; hence, their radii should increase. The outer radius in models vis 1 and vis 2 grows by a factor of $\sim 20$, while the outer radius in model vis 3 with the longer viscous timescale grows by a factor of $\sim 4$. Thus, gas disk radii as a function of class type and cluster age could directly test one of the main predictions of viscous disk models. In addition, gas disk radii, in combination with an estimate for the viscous timescale, constrain the average efficiency of angular momentum transport.

\subsection{Disk Wind}

An alternative scenario to consider is when angular momentum is not transported by turbulent viscosity but removed from the disk by a (MHD) wind (e.g., Blandford \& Payne 1982). While such a scenario can be motivated on theoretical grounds and from MHD simulations (e.g., Bai 2016), quantitative predictions on how mass accretion evolves with time and for a range of conditions are missing. In particular, it is not clear how (and if) the mass accretion rate depends on disk mass and stellar mass.

Therefore, in Appendix $\mathrm{C}$ we construct the simplest possible wind model where the mass accretion rate depends only on the (initial) strength of the magnetic field. Such a model can fit the observed scatter in the $M_{\text {dust }}-\dot{M}_{\text {acc }}$ plane, though we have to impose an additional stellar mass dependence in the mass accretion rate (in the $\alpha$-disk models, this dependence follows from the $M_{\text {dust }}-M_{\star}$ relation). The dependence of mass accretion rate on the wind properties is likely more complex (e.g., Armitage et al. 2013), and we stress that detailed predictions from MHD disk wind models are needed to test this scenario to the same degree as $\alpha$-disk models. The simple model is shown here only to illustrate how measurements of dust mass and mass accretion can be used to test and constrain these disk wind scenarios.

An additional observational constraint on disk wind models is the radial extent of the gas disk. Winds extract angular momentum from the system, and the disk can accrete without growing in size. Viscosity, on the other hand, redistributes angular momentum within the disk, and the disk grows in size when accreting. Measurements of the radial extent of the disk gas, in particular as a function of age, can provide key constraints on angular momentum transport in protoplanetary disks.

\subsection{Additional Physical Processes}

Several physical processes, not included in the constant- $\alpha$ disk model, could affect disk evolution and might contribute to the scatter in the observed $M_{\text {disk }}-\dot{M}_{\text {acc }}$ relation. The impact of various physical processes on the observed dust mass and mass accretion rate was investigated by Jones et al. (2012) and Rosotti et al. (2017). Following the analysis in the latter paper, we calculate a dimensionless accretion parameter, the ratio of accreted mass to disk mass, as a measure of the accretion efficiency in the disk. Figure 9 shows this ratio, here defined as

$$
\eta=\frac{\dot{M}_{\mathrm{acc}} t_{\mathrm{disk}}}{100 M_{\mathrm{dust}}},
$$

for disks in Lupus and Chamaeleon I (left panel) and for the constant- $\alpha$ disk model vis 1 (right panel). The dispersion in $\eta$ is estimated by linmix to be $0.8 \mathrm{dex}$, while the vis 1 model predicts a much smaller dispersion of 0.1 dex. The dispersion in $\eta$ contains similar diagnostic information to the stellar-massdetrended quantities, and the vis2 and vis3 models reproduce the observed dispersion in $\eta$.

The dispersion in $\eta$ can be increased by different physical processes in the following way:

1. Photoevaporation. Mass loss driven by stellar XUV photons becomes important for the disk structure when the mass accretion rate drops below the photoevaporation rate (e.g., Alexander et al. 2014). Jones et al. (2012) show that the $\eta$ increases only for a brief period at late times 
when the accretion rate is low, and this is unlikely to affect the dispersion in the majority of stars in our sample. External photoevaporation increases $\eta$ by an order of magnitude (Rosotti et al. 2017). While there are no massive stars near the low-mass star-forming regions Lupus and Chamaeleon I, external photoevaporation might play a role in these smaller clusters under certain conditions (e.g., Facchini et al. 2016; Haworth et al. 2017).

2. Layered Accretion and Dead Zones. In the presence of "dead zones," regions with low viscosity in the midplane of the disk, accretion may continue through well-ionized surface layers (Gammie 1996). The buildup of material at the edge of the dead zone may trigger disk instabilities that lead to enhanced episodes of accretion. Jones et al. (2012) show that layered accretion leads to a variation of orders of magnitude in $\eta$, but disks spend most of their time not accreting with only short outbursts of high accretion, which is unlikely to reproduce the observed distribution. Rosotti et al. (2017) suggest that a dead zone may lead to an $\eta$ below unity. Another complication with layered accretion is that it is unlikely to reproduce the stellar-mass dependence of mass accretion rates (Hartmann et al. 2006). We suggest that smaller variations in disk viscosity between surface layer and dead zone may produce a range in $\eta$ more consistent with what is observed, though constructing such a model is outside the scope of this paper. These variations would have to be significantly smaller than those typically assumed for a dead zone $\left(\alpha_{\text {layer }} / \alpha_{\text {dead }}<10^{3}-10^{4}\right)$ but large enough to have a significant effect on the mass accretion rate $(>10 ;$ see below), and could perhaps be of order $\alpha_{\text {layer }} / \alpha_{\text {dead }} \approx 10-100$.

3. Radial Variations in $\alpha$. MHD simulations of protoplanetary disks find that $\alpha$ can vary radially, although variations are not large outside the dead zone (see, e.g., Flock et al. 2011, 2017). Figure 1 in Rosotti et al. (2017) shows that variations of an order of magnitude in $\alpha$ have only a negligible impact on the long-term evolution of the disk.

4. Presence of Giant Planets. A giant planet forming in the disk may decrease the disk accretion rate by a factor of 4-10 if it is sufficiently massive (Lubow \& D'Angelo 2006) and decrease the accretion efficiency $\eta$ (Jones et al. 2012). Because giant planets are rare around Sunlike stars (10\%, Cumming et al. 2008) and even rarer around the low-mass stars in our sample (Johnson et al. 2010; Mulders et al. 2015), we do not expect giant planet formation to contribute significantly to the dispersion in $\eta$.

5. Grain Growth and Radial Drift. Grains that grow much larger than the wavelength where dust mass is estimated become undetectable, lowering $\eta$. Similarly, inward radial drift of dust grains reduces the detectable amount of dust in the outer disk, also lowering $\eta$ (e.g., Testi et al. 2014). Dust traps are crucial in preserving a detectable amount of dust grains at millimeter wavelengths (e.g., Pinilla et al. 2012). The variations in $\eta$ between disks due to grain growth and radial drift have not been quantified, but they would have to be of order $\sim 0.7$ dex to explain the observed scatter in dust mass.

\section{Conclusions}

We analyze the ALMA dust masses and X-Shooter mass accretion rates of protoplanetary disks in the $\sim 1-3 \mathrm{Myr}$ old Chamaeleon I and Lupus star-forming regions. We find the following:

1. The relation between dust mass, $M_{\text {dust }}$, and mass accretion rate, $\dot{M}_{\text {acc }}$, in Chamaeleon I has a slope consistent with linear of $0.8 \pm 0.2$ and a correlation coefficient of $r=0.6 \pm 0.1$. This result mirrors the findings in Lupus reported by Manara et al. (2016b).

2. There is significant scatter around the $M_{\text {dust }}-\dot{M}_{\text {acc }}$ relationship that is not predicted by viscously evolved disk models with a constant $\alpha$. The scatter around the best-fit $M_{\text {dust }}-M_{\star}$ relation, $\Delta M_{\text {dust }}$, and the scatter around the best-fit $\dot{M}_{\text {acc }}-M_{\star}$ relation, $\Delta \dot{M}_{\text {acc }}$, are only weakly correlated $(r \approx 0.3)$.

We simulate observations of an ensemble of evolving protoplanetary disks with a range of initial conditions using a Monte Carlo approach. Disk models where the viscosity is described by a constant $\alpha$ and with a viscous timescale shorter than the disk lifetime provide a good match to the observed $M_{\text {dust }}-M_{\star}$ and $\dot{M}_{\text {acc }}-M_{\star}$ relations. However, the predicted correlations between $M_{\text {dust }}-\dot{M}_{\text {acc }}$ and $\Delta M_{\text {dust }}-\Delta \dot{M}_{\text {acc }}$ are too tight $(r>0.9)$ to be consistent with the Lupus-Chamaeleon I data set. We find two possible solutions:

1. The scatter in observed dust mass and mass accretion rate does not reflect a dispersion in disk initial conditions (mass, disk, $\alpha$, age). In this scenario, the observed scatter must arise from additional physical processes: most likely grain growth and radial drift affect the observable dust mass, while variability on large timescales affects the mass accretion rates. These processes should introduce variations in the dust-to-gas ratio between disks with a standard deviation of $0.7 \mathrm{dex}$ and time variability in the accretion rate with a standard deviation of 0.7 dex, much larger than the dispersion in initial disk mass $(0.3 \mathrm{dex})$.

2. Disks do not evolve substantially at the age of Lupus and Chamaeleon I owing to a low viscosity $(\alpha \sim 0.001)$ or large initial disk radius $\left(R_{\text {disk }}>100 \mathrm{au}\right)$. A large dispersion in these two parameters creates scatter in the observed mass accretion rates that is not correlated with the scatter in the observed (and initial) disk mass, matching the observed weak correlation between $\Delta M_{\text {dust }}-\Delta \dot{M}_{\text {acc }}$. See also Lodato et al. (2017).

The large discrepancy between the observables and gas disk properties indicates that dust mass and mass accretion rate may be imperfect tracers of disk evolution. More accurate estimates of the disk mass, for example, with spatially resolved multiwavelength observations with ALMA, and of the size of gaseous disks are critical to test different evolutionary models.

It is also possible that a different source of angular momentum transport, such as MHD disk winds, may drive accretion in protoplanetary disks. While we show that alternate mechanisms can be consistent with the observed correlation between disk mass and mass accretion rate discussed here and in Manara et al. (2016b), quantitative predictions on how winddriven mass accretion rates scale with disk and stellar properties are needed to test this scenario. 


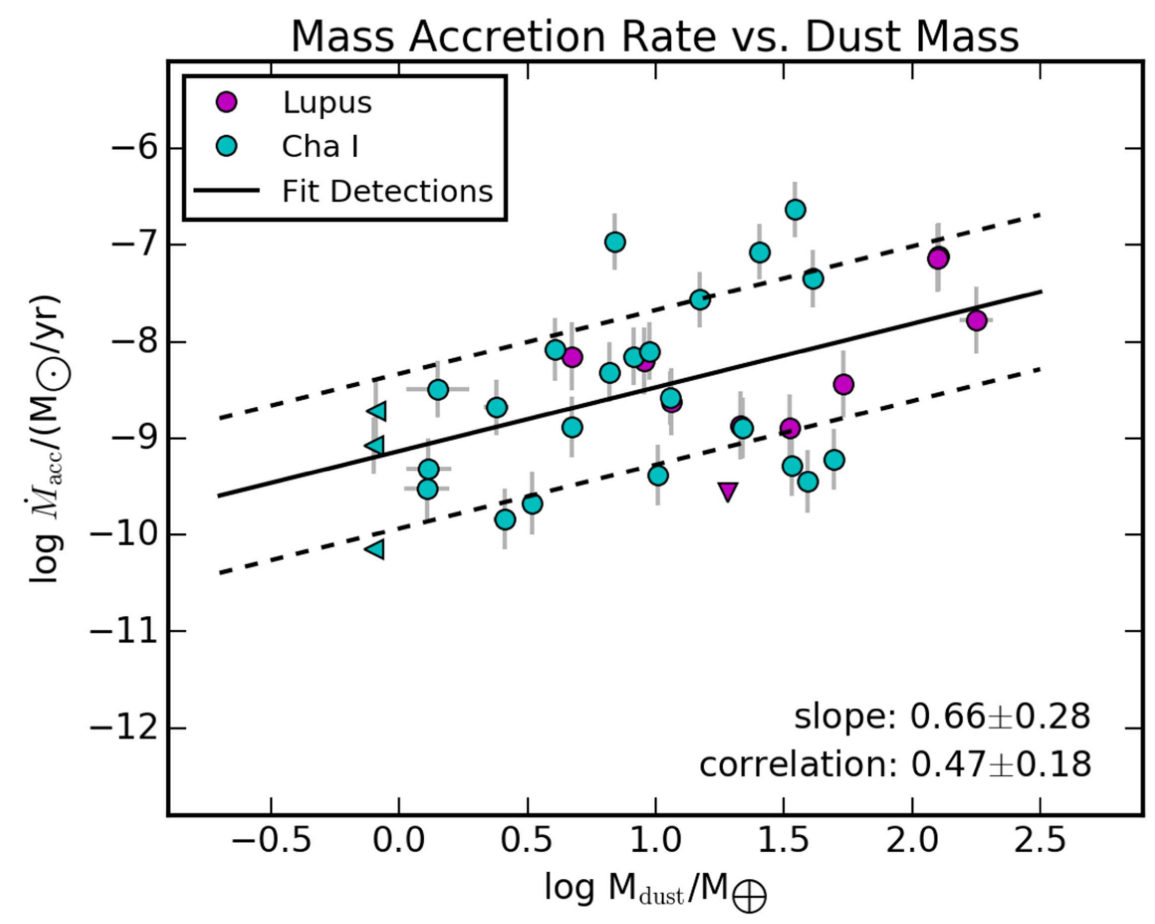

Figure 10. Dust masses vs. mass accretion rates for a restricted stellar mass range, $0.5 M_{\odot} \leqslant M_{\star} \leqslant 1.0 M_{\odot}$. The black lines show the best-fit regression curve (solid line) and $1 \sigma$ dispersion (dotted line).

Software: linmix (Kelly 2007), NumPy (van der Walt et al. 2011), Matplotlib (Hunter 2007).

We are grateful to the anonymous referee for a constructive review that has improved the quality of the paper. We thank Kaitlin Kratter, Kees Dullemond, Paola Pinilla, and Mario Flock for helpful comments. This material is based on work supported by the National Aeronautics and Space Administration under Agreement no. NNX15AD94G for the program Earths in Other Solar Systems. The results reported herein benefited from collaborations and/or information exchange within NASA's Nexus for Exoplanet System Science (NExSS) research coordination network sponsored by NASA's Science Mission Directorate. I.P. acknowledges support from an NSF Astronomy \& Astrophysics Research Grant (ID: 1515392).

\section{Appendix A \\ Limited Stellar Mass Range}

The analysis of disk properties over a wide range of stellar masses may be impacted by systematic uncertainties in stellar evolutionary models or more complex stellar-mass dependencies that are not accounted for in this work. In particular, derived stellar masses suffer from larger uncertainties toward the lower-mass end. On the other hand, mass accretion rates of stars more massive than a solar mass may be less reliable. In addition, Alcalá et al. (2017) and Manara et al. (2017) find tentative evidence for a different slope in the $\dot{M}_{\mathrm{acc}}-M_{\star}$ distribution at lower stellar masses. To assess the influence of the uncertainties described above, we re-evaluate the strength of the $\dot{M}_{\text {acc }}-M_{\text {dust }}$ correlation for a limited range in stellar masses $\left(0.5-1.0 M_{\odot}\right)$, where the stellar masses and mass accretion rates are most reliable.

Figure 10 shows the mass accretion rates of the combined sample as a function of dust mass for this limited stellar mass range. We fit a moderate correlation $(r=0.5 \pm 0.2)$ with a slope that is flatter than linear $(0.7 \pm 0.3)$ but compatible within errors. The confidence intervals on these parameters are larger owing to the lower number of stars included in the analysis. Within errors, the correlation is consistent with the $\dot{M}_{\text {acc }}-M_{\text {dust }}$ correlation for the entire sample. There is no evidence of a tight linear correlation between $M_{\text {dust }}$ and $\dot{M}_{\text {acc }}$ in this restricted stellar mass range. Hence, we conclude that there is no evidence that combining disk properties over a wide range of stellar masses reduces the strength of the observed correlations.

\section{Appendix B Accretion Variability Model}

The measured mass accretion rate is known to vary on timescales of hours to years with a typical magnitude of $\sim 0.4$ dex (Costigan et al. 2012, 2014; Venuti et al. 2014). This variability would introduce additional scatter in the $\dot{M}_{\mathrm{acc}}-M_{\star}$ relation that could weaken the correlation between dust mass and mass accretion rate. Processes like grain growth and radial drift (e.g., Birnstiel \& Andrews 2014) and disk mass loss (e.g., Gorti et al. 2015; Bai 2016) change the dust-to-gas ratio of the disk and are a potential source of scatter in the observed dust masses.

We run a set of constant- $\alpha$ disk models, vis 2 , with a deviation between the instantaneous mass accretion rate and the disk mass accretion rate of $f_{\mathrm{acc}}=0.4 \mathrm{dex}$, consistent with the observed variability. This model requires a lower dispersion in initial disk mass of 0.6 dex to fit the observed scatter in $\dot{M}_{\text {acc }}$. Because the lower dispersion in initial disk mass reduces the observable scatter in the observed dust mass as well, we introduce a dispersion in the gas-to-dust ratio of $f_{\mathrm{gtd}}$ of $0.4 \mathrm{dex}$ to fit the data. The top two panels of Figure 11 show that the simulated $M_{\text {dust }}-M_{\star}$ and $\dot{M}_{\text {acc }}-M_{\star}$ relations are consistent with the observations.

The added variability weakens the inferred correlation between $M_{\text {dust }}$ and $\dot{M}_{\text {acc }}(r=0.8)$ but remains more tightly correlated than observed $(r=0.6)$. The difference is significant at the $2.6 \sigma$ level. A strong correlation $(r=0.7)$ remains present 

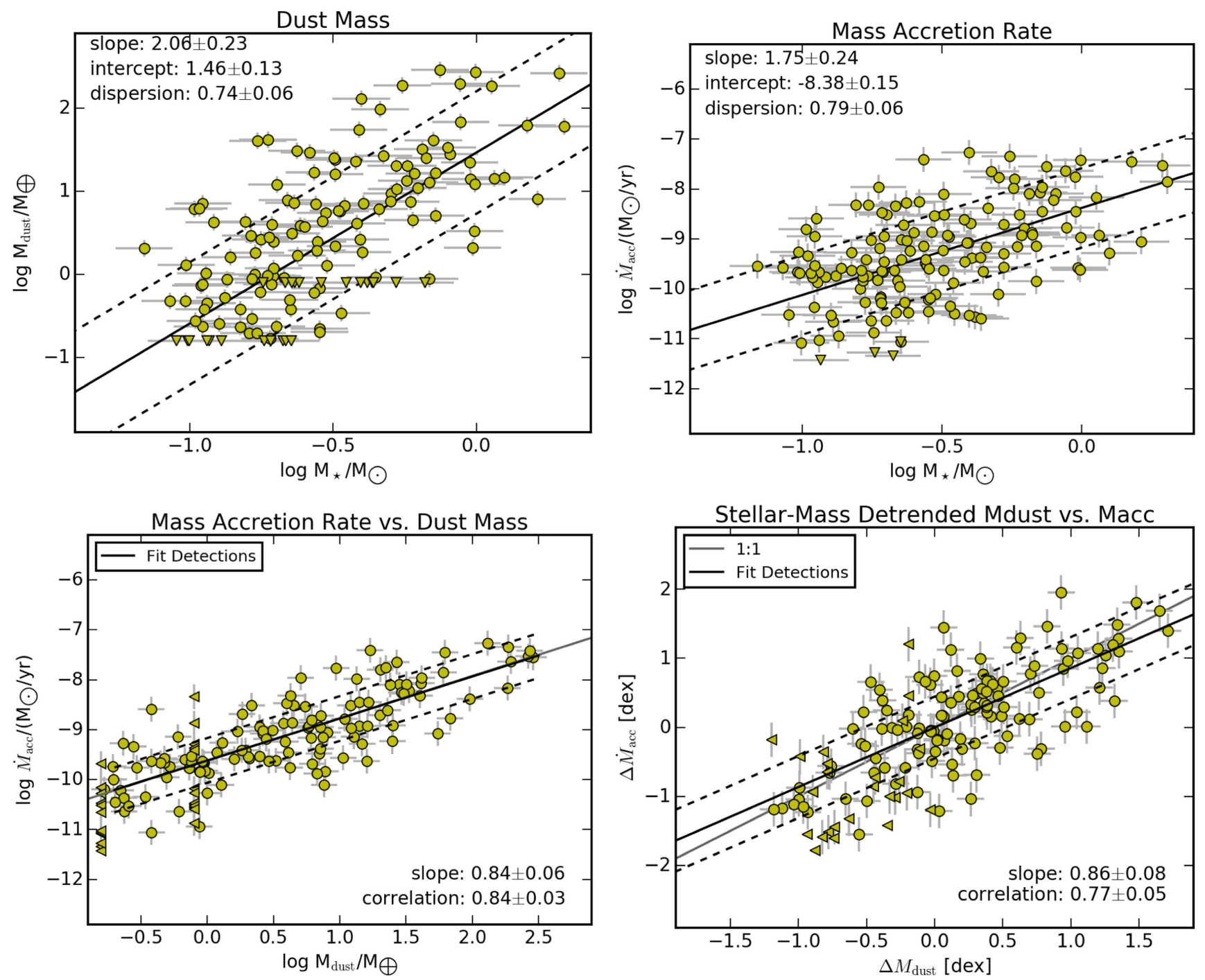

Figure 11. Synthetic observations for a disk model with a accretion rate variability consistent with that observed on timescales up to years. Same panels as in Figure 6 .

after detrending, indicating that the magnitude of the observed accretion rate variability on short timescales is not sufficient to erase a correlation between disk mass and mass accretion rate in the observables.

\section{Appendix C \\ Disk Wind Model}

An alternative approach to explaining the weak correlation between $M_{\text {dust }}$ and $\dot{M}_{\text {acc }}$ is assuming an evolutionary model where the mass accretion rate is not dependent on the local disk properties. This approach is motivated by recent theoretical and observational findings that nonviscous processes such as disk winds may play an important role in disk evolution as discussed in the introduction. There are, however, no quantitative predictions from MHD disk wind models of how mass accretion rate scales with stellar and disk properties. Hence, we devise a "thought experiment" in which we explore the observational signatures of a disk that accretes through an angular momentum transport mechanism other than a turbulent viscosity. The underlying assumption is that the (unknown) strength of the magnetic field, which varies from disk to disk, determines the mass accretion rate.
Table 3

Initial Conditions for the Simulated Disk Wind Model

\begin{tabular}{lcc}
\hline \hline Parameter (Unit) & Value & Dispersion (dex) \\
\hline$M_{\text {disk }, 0}\left(M_{\odot}\right)$ & $0.02 M_{\star}^{1.9}$ & 1.0 \\
$t_{\text {disk }}(\mathrm{Myr})$ & 2.0 & 0.3 \\
$\dot{M}_{\text {acc }}\left(M_{\odot} \mathrm{yr}^{-1}\right)$ & $10^{-8} M_{\star}^{1.9}$ & 0.8 \\
\hline
\end{tabular}

We construct a toy model where the mass accretion rate is independent of the conditions in the disk. The time evolution of the disk is described by

$$
M_{\mathrm{disk}}=M_{\mathrm{disk}, 0}-t \dot{M}_{\mathrm{acc}} \text {. }
$$

This assumption is loosely based on the constant magnetic flux model in Bai (2016), in which the mass accretion rate remains constant for $\sim 2 \mathrm{Myr}$ while the disk decreases in mass. This assumption can be relaxed, as it is not necessarily consistent with the observed decrease in mass accretion rate with age (e.g., Sicilia-Aguilar et al. 2010; Antoniucci et al. 2014; Hartmann et al. 2016). The disk is assumed to be dispersed if $M_{\text {disk }}<0$. Disks with a larger initial mass accretion rate 

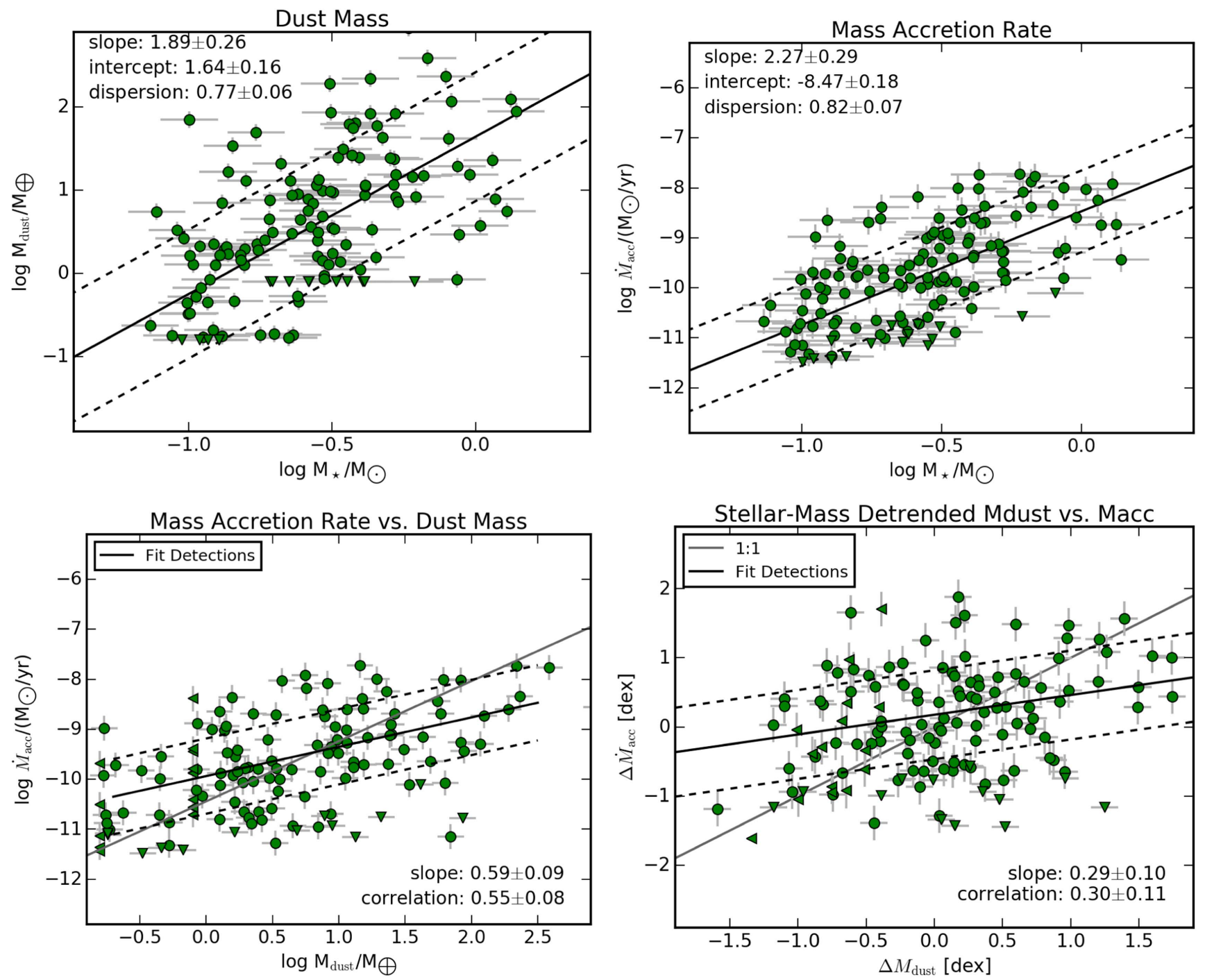

Figure 12. Synthetic observations for the disk wind model described in the text. Same panels as in Figure 6.

disperse their disks faster, leading to a lower disk mass when observed. The model has three main free parameters. In particular, the mass accretion rate is unconstrained by the choice of disk mass, and stellar-mass dependency has to be introduced separately in the initial disk mass and mass accretion rate to fit the observed $M_{\mathrm{dust}}-M_{\star}$ and $\dot{M}_{\mathrm{acc}}-M_{\star}$ relations. The free parameters in this model, $M_{\mathrm{disk}, 0}, t$, and $\dot{M}_{\mathrm{acc}}$, are assumed to follow a lognormal distribution. The mean disk age, $t=2 \mathrm{Myr}$, and dispersion, $0.3 \mathrm{dex}$, are the same as in the $\alpha$-disk model for consistency. The mean and standard deviations of the remaining two free parameters, $M_{\text {disk, } 0}$ and $\dot{M}_{\text {acc }}$, are chosen to reproduce the observed stellar-mass dependencies and scatter in $M_{\text {dust }}$ and $\dot{M}_{\text {acc }}$ and listed in Table 3.

We conduct synthetic observations in the same manner as for the $\alpha$-disk models, perturbing $M_{\star}, M_{\text {dust }}$, and $\dot{M}_{\text {acc }}$ with their respective observational uncertainties. We increase the sample size to 250 disks such that 140 disks remain at the time of observation. The initial disk mass, $M_{\text {disk,0, }}$, and mass accretion rate, $\dot{M}_{\text {acc }}$, are constrained by comparing the model to the observed dust mass and mass accretion rates. Note that both parameters have a stellar-mass dependency of $M_{\star}^{1.9}$, whereas in the $\alpha$-disk model only the initial disk mass is assumed to be stellar mass dependent. The initial distribution of disk mass accretion rates, $\dot{M}_{\mathrm{acc}}$, has a dispersion of 1.0 dex. This yields an observable dispersion in mass accretion rates of 0.8 dex after 2 Myr (consistent with the observed scatter around the $\dot{M}_{\text {acc }}-M_{\star}$ relation) and a dispersion in dust disk mass of $\sim 0.3$ dex (significantly smaller than the observed scatter of $0.8 \mathrm{dex}$ ). The additional scatter can be accounted for by introducing a dispersion in initial disk mass, $M_{\text {disk, } 0}$, of 0.7 dex.

The wind model, shown in Figure 12, produces a moderate correlation between dust mass and mass accretion rate of $0.6 \pm 0.1$, consistent with the observations. The inferred slope of the $M_{\text {dust }}-\dot{M}_{\text {acc }}$ relation, $0.7 \pm 0.1$, is consistent with the slope derived from the observed data and from model vis3. The detrended quantities $\Delta M_{\text {dust }}$ and $\Delta \dot{M}_{\text {acc }}$ show a weak correlation, $r=0.3 \pm 0.1$, again consistent with the observed correlation. No variations in the dust-to-gas ratio or accretion rate variability are required to fit the data.

\section{ORCID iDs}

Gijs D. Mulders (1D https://orcid.org/0000-0002-1078-9493

Ilaria Pascucci (1) https://orcid.org/0000-0001-7962-1683 
Carlo F. Manara (ํ) https://orcid.org/0000-0003-3562-262X

Leonardo Testi 1 ib https://orcid.org/0000-0003-1859-3070

Gregory J. Herczeg (ํ) https://orcid.org/0000-0002-7154-6065

Thomas Henning (10 https://orcid.org/0000-0002-1493-300X

Subhanjoy Mohanty (1) https://orcid.org/0000-0002-

9896-8313

\section{References}

Alcalá, J., Manara, C. F., Natta, A., et al. 2017, A\&A, 600, A20

Alcalá, J., Natta, A., Manara, C. F., et al. 2014, A\&A, 561, A2

Alexander, R., Pascucci, I., Andrews, S., Armitage, P., \& Cieza, L. 2014,

Protostars and Planets VI (Tucson, AZ: Univ. Arizona Press), 475

Alexander, R. D., \& Armitage, P. J. 2006, ApJL, 639, L83

Alexander, R. D., Clarke, C. J., \& Pringle, J. E. 2006, MNRAS, 369, 229

Andrews, S., Rosenfeld, K. A., Kraus, A. L., \& Wilner, D. J. 2013, ApJ, 771,129

Andrews, S., Wilner, D. J., Hughes, A. M., Qi, C., \& Dullemond, C. P. 2010, ApJ, 723, 1241

Ansdell, M., Williams, J. P., van der Marel, N., et al. 2016, ApJ, 828, 46

Antoniucci, S., Garcia Lopez, R., Nisini, B., et al. 2014, A\&A, 572, A62

Armitage, P. J. 2011, ARA\&A, 49, 195

Armitage, P. J., Clarke, C. J., \& Palla, F. 2003, MNRAS, 342, 1139

Armitage, P. J., Simon, J. B., \& Martin, R. G. 2013, ApJL, 778, L14

Bai, X.-N. 2016, ApJ, 821, 80

Bai, X.-N., \& Stone, J. M. 2013, ApJ, 769, 76

Balbus, S. A.. \& Hawley, J. F. 1991, ApJ, 376, 214

Baraffe, I., Homeier, D., Allard, F., \& Chabrier, G. 2015, A\&A, 577, A42

Beckwith, S. V. W., Henning, T., \& Nakagawa, Y. 2000, in Protostars and Planets IV, ed. V. Mannings, A. P. Boss, \& S. S. Russell (Tucson: Univ. Arizona Press), 533

Birnstiel, T., \& Andrews, S. 2014, ApJ, 780, 153

Birnstiel, T., Klahr, H., \& Ercolano, B. 2012, A\&A, 539, A148

Blandford, R. D., \& Payne, D. G. 1982, MNRAS, 199, 883

Caratti, O., Caratti, A., Garcia Lopez, R., et al. 2012, A\&A, 538, A64

Chabrier, G. 2003, ApJL, 586, L133

Chambers, J. E. 2009, ApJ, 705, 1206

Costigan, G., Scholz, A., Stelzer, B., et al. 2012, MNRAS, 427, 1344

Costigan, G., Vink, J. S., Scholz, A., Ray, T., \& Testi, L. 2014, MNRAS, 440,3444

Cridland, A. J., Pudritz, R. E., Birnstiel, T., Cleeves, L. I., \& Bergin, E. A. 2017, MNRAS, 469, 3910

Cumming, A., Butler, R. P., Marcy, G. W., et al. 2008, PASP, 120, 531

Draine, B. T. 2006, ApJ, 636, 1114

Dullemond, C. P., Natta, A., \& Testi, L. 2006, ApJL, 645, L69

Ercolano, B., Mayr, D., Owen, J. E., Rosotti, G., \& Manara, C. F. 2014, MNRAS, 439, 256

Ercolano, B., \& Pascucci, I. 2017, RSOS, 4, 170114

Facchini, S., Clarke, C. J., \& Bisbas, T. G. 2016, MNRAS, 457, 3593

Fang, M., Kim, J. S., van Boekel, R., et al. 2013, ApJS, 207, 5

Fang, M., van Boekel, R., Wang, W., et al. 2009, A\&A, 504, 461

Fedele, D., van den Ancker, M. E., Henning, T., Jayawardhana, R., \& Oliveira, J. M. 2010, A\&A, 510, A72

Feiden, G. A. 2016, A\&A, 593, A99

Flaherty, K. M., Hughes, A. M., Rose, S. C., et al. 2017, ApJ, 843, 150

Flock, M., Dzyurkevich, N., Klahr, H., Turner, N. J., \& Henning, T. 2011, ApJ, 735,122

Flock, M., Fromang, S., González, M., \& Commerçon, B. 2013, A\&A, 560, A 43

Flock, M., Fromang, S., Turner, N. J., \& Benisty, M. 2017, ApJ, 835, 230

Gammie, C. F. 1996, ApJ, 457, 355

Gorti, U., Hollenbach, D., \& Dullemond, C. P. 2015, ApJ, 804, 29
Gressel, O., Turner, N. J., Nelson, R. P., \& McNally, C. P. 2015, ApJ, 801, 84 Hartmann, L. W., Calvet, N., Gullbring, E., \& D’Alessio, P. 1998, ApJ, 495,385

Hartmann, L. W., D’Alessio, P., Calvet, N., \& Muzerolle, J. 2006, ApJ, 648,484

Hartmann, L. W., Herczeg, G., \& Calvet, N. 2016, ARA\&A, 54, 135

Haworth, T. J., Facchini, S., Clarke, C. J., \& Cleeves, L. I. 2017, MNRAS, 468, L108

Hendler, N. P., Mulders, G. D., Pascucci, I., et al. 2017, ApJ, 841, 116

Hunter, J. D. 2007, CSE, 9, 90

Johnson, J. A., Aller, K. M., Howard, A. W., \& Crepp, J. R. 2010, PASP, 122,905

Jones, M. G., Pringle, J. E., \& Alexander, R. D. 2012, MNRAS, 419, 925

Kalyaan, A., Desch, S. J., \& Monga, N. 2015, ApJ, 815, 112

Kelly, B. C. 2007, ApJ, 665, 1489

Kratter, K., \& Lodato, G. 2016, ARA\&A, 54, 271

Kunz, M. W., \& Lesur, G. 2013, MNRAS, 434, 2295

Lodato, G., Scardoni, C. E., Manara, C. F., \& Testi, L. 2017, MNRAS, in press (arXiv:1708.09467)

Lubow, S. H., \& D'Angelo, G. 2006, ApJ, 641, 526

Lynden-Bell, D., \& Pringle, J. E. 1974, MNRAS, 168, 603

Malygin, M. G., Klahr, H., Semenov, D., Henning, T., \& Dullemond, C. P. 2017, arXiv:1704.06786

Mamajek, E. E. 2009, in AIP Conf. Proc. Exoplanets and Disks: Their Formation and Diversity: Proc. Int. Conf., ed. T. Usuda, M. Tamura, \& M. Ishii (New York: AIP), 3

Manara, C. F., Fedele, D., Herczeg, G. J., \& Teixeira, P. S. 2016a, A\&A, 585, A136

Manara, C. F., Rosotti, G., Testi, L., et al. 2016b, A\&A, 591, L3

Manara, C. F., Testi, L., Herczeg, G. J., et al. 2017, arXiv:1704.02842

Manara, C. F., Testi, L., Natta, A., et al. 2014, A\&A, 568, A18

Mendigutía, I., Brittain, S., Eiroa, C., et al. 2013, ApJ, 776, 44

Mohanty, S., Ercolano, B., \& Turner, N. J. 2013, ApJ, 764, 65

Mordasini, C., Alibert, Y., \& Benz, W. 2009, A\&A, 501, 1139

Mulders, G. D., Pascucci, I., \& Apai, D. 2015, ApJ, 814, 130

Natta, A., Testi, L., \& Randich, S. 2006, A\&A, 452, 245

Nguyen, D. C., Scholz, A., van Kerkwijk, M. H., Jayawardhana, R., \& Brandeker, A. 2009, ApJL, 694, L153

Nisini, B., Antoniucci, S., Giannini, T., \& Lorenzetti, D. 2005, A\&A, 429, 543

Owen, J. E., Ercolano, B., \& Clarke, C. J. 2011, MNRAS, 412, 13

Pascucci, I., Testi, L., Herczeg, G. J., et al. 2016, ApJ, 831, 125

Pinilla, P., Birnstiel, T., Ricci, L., et al. 2012, A\&A, 538, A114

Pringle, J. E. 1981, ARA\&A, 19, 137

Rafikov, R. R. 2017, ApJ, 837, 163

Ricci, L., Testi, L., Natta, A., et al. 2010, A\&A, 512, A15

Rice, K. 2016, PASA, 33, e012

Rosotti, G. P., Clarke, C. J., Manara, C. F., \& Facchini, S. 2017, MNRAS, 468,1631

Shakura, N. I., \& Sunyaev, R. A. 1973, A\&A, 24, 337

Sicilia-Aguilar, A., Henning, T., \& Hartmann, L. W. 2010, ApJ, 710, 597

Siess, L., Dufour, E., \& Forestini, M. 2000, A\&A, 358, 593

Stepinski, T. F. 1998a ApJ, 507, 361

Stepinski, T. F. 1998b Icar, 132, 100

Teague, R., Guilloteau, S., Semenov, D., et al. 2016, A\&A, 592, A49

Testi, L., Birnstiel, T., Ricci, L., et al. 2014, Protostars and Planets VI (Tucson, AZ: Univ. Arizona Press), 339

Turner, N. J., Fromang, S., Gammie, C., et al. 2014, Protostars and Planets VI (Tucson, AZ: Univ. Arizona Press), 411

van der Marel, N., Van Dishoeck, E. F., Bruderer, S., et al. 2013, Sci, 340,1199

van der Walt, S., Colbert, S. C., \& Varoquaux, G. 2011, CSE, 13, 22

Venuti, L., Bouvier, J., Flaccomio, E., et al. 2014, A\&A, 570, A82

Venuti, L., Bouvier, J., Irwin, J., et al. 2015, A\&A, 581, A66

White, R. J., \& Hillenbrand, L. A. 2004, ApJ, 616, 998 